\title{
Geomorfología y cambio ambiental en el entorno de los yacimientos púnicos de la Ilanura de Terralba (Golfo de Oristano, isla de Cerdeña, Italia)
}

\author{
J.M. Ruiz(1), P. Carmona(1), C. Gómez-Bellard ${ }^{(2)}$ y P. van Dommelen ${ }^{(3)}$ \\ (1) Departamento de Geografía, Universidad de Valencia, Avda. Blasco Ibáñez 28, E-46010 Valencia, España \\ jose.m.ruiz-perez@uv.es; pilar.carmona@uv.es \\ (2) Departamento de Arqueología, Universidad de Valencia, Avd. Blasco Ibáñez 28, E-46010 Valencia, España \\ Bellard@uv.es \\ (3) Joukowsky Institute for Archaeology and the Ancient World, Brown University, Box 1837 / 60 George Street, \\ Providence, RI 02912, USA \\ peter_van_dommelen@brown.edu
}

\begin{abstract}
RESUMEN
Se presenta un estudio geomorfológico de la llanura de Terralba (costa SW de la Isla de Cerdeña, Italia) con el objeto de contextualizar la alta concentración de asentamientos rurales de época púnica en torno a dicha población y examinar los cambios ambientales. La metodología se basa en el análisis geomorfológico de detalle y en el estudio de registros sedimentarios de diferentes ambientes en cortes artificiales y sondeos (cronología a partir de restos arqueológicos y dataciones 14C). El trabajo se completó con información de diversas fuentes cartográficas, datos históricos y geoarqueológicos. Los yacimientos púnicos se localizan sobre una amplia llanura aluvial, modelada por mantos arenosos y humedales, entre los llanos de inundación de los ríos Sitzerri-Mannu y Mogoro. Las unidades eólicas, holocenas y pleistocenas, son el elemento más característico de la geomorfología del área. Los resultados aportan información acerca del contexto ambiental de la colonización púnica, al tiempo que se hallaron evidencias de significativos cambios geomorfológicos y ambientales durante más de dos milenios. Estos cambios afectaron a la línea de costa, lagoons, zonas palustres y a los ambientes fluviodeltaico y eólico. El afloramiento de agua dulce en los "paulis" o pequeñas depresiones intercaladas entre mantos arenosos, bien drenados y con suelos adecuados para el cultivo, debió ser un factor importante en el asentamiento de las granjas púnicas.
\end{abstract}

Palabras clave: cambio ambiental, mantos arenosos, geoarqueología, geomorfología litoral, Holoceno.

\section{Geomorphology and environmental change around the Punic sites of the Terralba plain (Oristano Gulf, Sardinia, Italy)}

\begin{abstract}
We report a geomorphological study of the coastal plain of Terralba (SW coast of the island of Sardinia, Italy) in order to contextualize the high concentration of Punic rural settlements and analyze the environmental changes. The methodology is based on a detailed geomorphological study and sedimentological analysis of several records from different environments in artificial cuts and cores (chronology from archaeological remains and $14 \mathrm{C}$ dating). The study was also completed with information from various cartographic sources, and historical and geoarchaeological data. The Punic sites are located on a wide alluvial plain, modelled by aeolian sand mantles and wetlands, between the floodplain of the Sitzerri-Mannu and Mogoro rivers. Extensive aeolian units (Holocene and Pleistocene) are the most characteristic features of the geomorphology of the area. The results provide useful information on the environmental context of the Punic settlements on the coastal plain of Terralba, while evidence of significant geomorphological and environmental changes were found for over two millennia affecting the coastline, lagoon, marsh, aeolian and fluviodeltaic environments. The emergence of fresh water in the "paulis" or small depressions interspersed between dunes and well-drained soils suitable for cultivation have been a factor in the settlement of Punic farms.
\end{abstract}

Key words: coastal geomorphology, dunes, environmental change, geoarchaeology, Holocene. 
Ruiz, J.M., et al., 2018. Geomorfología y cambio ambiental en el entorno de los... Boletín Geológico y Minero, 129 (1/2): $331-351$

\section{ABRIDGED ENGLISH VERSION}

\section{Introduction}

Recent geoarchaeological research has improved our knowledge of Mediterranean coastal environmental changes in the Holocene (Marriner and Morhange, 2007; Anthony et al., 2014; Morhange et al., 2015). Around the town of Terralba (SE Sardinia), archaeological surveys detected a large concentration of rural settlements dating from Punic and Roman times (Van Dommelen, 1998). The farms were mainly located over sandy mounds, with soil suitable for fruit and olive growing (Nicosia et al., 2013). The aims of our study are to characterise the physical environment of these deposits and analyse the environmental changes occurring in their surroundings (Fig 1).

\section{Materials and methods}

A detailed geomorphological study was carried out, describing the stratigraphy of manual surveys (Eijkelkamp probe) and sections in different sub-environments and to perform $14 \mathrm{C}$ dating. Mapping of the Autonomous Region of Sardinia were used, including a DEM Lidar (1 $\mathrm{m}$ resolution), aerial orthophotos from 1954 and geological maps. In addition, we analysed historical maps and plans from the "Società Bonifiche Sarde" (Arborea), which allowed us to recognise recent artificial changes. All geographical data were entered in a GIS (ArcGIS and Microstation V8).

\section{Regional setting of the study area}

The study area encompasses the plain around Terralba (9 m s.n.m.), approximately $10.5 \mathrm{~km}$ from the Gulf of Oristano (northern Campidano). The Campidano half-graben is limited by a fault (NW-SE) to the east of Cabo di San Marco (Fais et al., 1996) and bordered by two granite-metamorphic horsts. During the Tertiary, several phases of andesitic volcanism occurred and today there is a relative seismic calm (Angelone et al., 2005). The plain presents alternate (Pleistocene and Holocene) marine, continental and transition materials (coastal lagoons and marshy areas) (Fig. 2 and 3). During the agrarian transformation of "Sardinian Land Reclamation" (around 1930) the dunes were razed and many lakes and ponds dried out (among them the Sassu Lagoon).

\section{Results}

Coastal morphology

The Oristano Gulf micro-tidal coast consists of a beach of sands deposited by the river Tirso (the largest in Sardinia) and from the adjacent shallow marine shelf. The littoral strand with dunes extends to the Corru Mannu point, isolating back-barrier lagoons of elongated morphology from the sea (Figs. 4 and 5).

\section{Fluvial system}

Two main fluvial valleys are located around Terralba. To the south, the Mannu-Sitzerri river valley forms an inland delta in the Stagno di Santa Maria (alongside Neapolis) and, to the west, the Mogoro river valley which, before being artificially diverted, forked into several channels that drained into the Stagno di Sassu (Fig. 4).

\section{Dune system}

Holocene and Pleistocene eolian units extend around the towns of Arborea, Terralba and San Nicolò d'Arcidano, razed during Mussolini's "Land Reclamation" (Soru, 2000). However, the DEM Lidar distinguishes dune ridges parallel to the coast and dune mantles formed by strong winds from the NW ("maestrali") extending some kilometres inwards (Fig. 4, 5, 6 and 7).

\section{Lagoon and wetland environments}

The coastal plain includes several brackish to hypersaline coastal lagoons and many small and medium-sized marshy areas ("pauli"), whose morphology, size and processes are varied. The larger ones have elongated 
Ruiz, J.M., et al., 2018. Geomorfología y cambio ambiental en el entorno de los... Boletín Geológico y Minero, 129 (1/2): $331-351$

morphology and are hundreds of metres in length. Other "pauli" (tens of metres) are found among the elongated dune ridges (NW-SE). Finally, there is another type of inland "pauli" (around Terralba) with montmorillonite clay outcrops, set between narrow dune alignments and which may be interpreted as areas of wind deflation (Fig. 4, 5, 6 and 8).

\section{Environmental change records in recent millennia}

Pauli sa Ussa environment

Near Pauli sa Ussa there is an artificial section (P3B) 2 metres thick. Three levels are distinguished, interpreted as (a) a lagoon associated with Pauli sa Ussa; (b) a Punic-era eolian environment on the bank of a freshwater lagoon or marsh; c) loose sands that seal off the wetland. At the level (b) composed of dark grey sand with carbonised plant remains and gastropods Lymnaea palustris, a shell was dated by AMS $14 \mathrm{C}(490-460 \mathrm{cal}$ yr BC / 420 to 390 years cal B.C.), a date consistent with the ceramic fragments ascribed to the Punic era (Fig. 9; Table 1).

Record of the Mogoro river lagoon delta

A survey (P26) with Eijkelkamp probe was carried out in the delta plain of the Mogoro river (1.3 m s.n.m.) at the di Sassu lagoon (N ofTerralba). A sandy mud layer with Cerastoderma sp. (brackish lagoon) was reached, dated by $14 C$ (AMS) to 800-760 cal years BC / 680-670 cal yr BC to $3.5 \mathrm{~m}$ deep and 1010-890 cal yr BC / 870$850 \mathrm{cal}$ yrs $B C$ at $4 \mathrm{~m}$ depth. An overlying sludge layer (2-3 $\mathrm{m}$ deep) with aquatic vegetation and Planorbis $s p$. fills a freshwater lagoon or marsh (Fig. 10; Table 1).

Record of the S.M. di Neapolis marsh

A manual survey in the marshes near the site of Santa Maria di Neapolis (1.7 m s.n.m.) reached a depth of 4.2 $m$. The upper part of the sedimentary column comprises distal contributions from the Mannu-Sitzerri river in a marshy environment, while coarse basal sands were deposited in the former estuary of Marceddi-San Giovanni (Fig. 11).

\section{Discussion}

Dunes and wetlands: an environment subject to Punic-era agrarian colonisation

The eolian mantle of Arborea-Terralba would be formed from the rapid sea-level fall, when huge quantities of sand were left exposed. This eolian episode was dated at around $75 \mathrm{ka} B P$ in cliffs of the Sardinian west coast (Andreucci et al., 2010) and is contemporary to the UO unit at El Abalorio on the Atlantic coast of Huelva (Zazo et al., 2011). We have limited data on Holocene eolian phases in Terralba, although the upper layer contains abundant archaeological remains. In the margin of Pauli sa Ussa, a $1 \mathrm{~m}$ thick layer of sand covers the Punic era levels. At the Pauli Stincus site, the layer is from several decimetres to $1.2 \mathrm{~m}$ on Pleistocene fluvial material (Nicosia et al., 2013) and several decimetres on Pleistocene dunes at Bau Arena. The deforestation associated with the Punic farms would have triggered episodes of eolian reactivation. For reference, the eolian accumulation on the Andalusian Atlantic coast occurred after 2,700 cal BP, when the progradation of spits and associated dunes began (Borja et al., 1999; Zazo et al., 2011).

Coastline changes: infilling of the lagoons and progradation of the Tirso river delta

The dated deltaic record of the Mogoro river shows that the di Sassu lagoon used to extend almost to Terralba in the Phoenician-Punic era, which would make a port or jetty lagoon possible. The abundance of Cerastoderma sp. at the core base indicates an open lagoon, before progradation of the most recent littoral spit.

The inputs of the Tirso river Holocene delta feed coastal spits and dune ridges, increasingly advancing seawards. A nuraghe (Bronce Age), at 2,500 $\mathrm{m}$ from the coast, provides a minimum date for progradation. These changes are consistent with those described in many ancient Mediterranean sea or lagoon ports, which were left landlocked as a result of deltaic progradation (Morhange et al., 2015). 
Ruiz, J.M., et al., 2018. Geomorfología y cambio ambiental en el entorno de los... Boletín Geológico y Minero, 129 (1/2): $331-351$

\section{Conclusions}

Data on the environmental context of Punic colonisation in the coastal plain of Terralba were provided, while evidence was found of geomorphological and environmental changes for more than two millennia. Notable features included the development of coastal spits, the gradual filling in of lagoons by inland deltas and windblown sand redistribution, which contributed to the infill of wetlands ("paulis") in the Terralba plain.

From the geoarchaeological viewpoint, the high concentration of Punic farms on dunes in Terralba can be explained by local edaphic (Nicosia et al., 2011) and hydrogeological factors (freshwater availability in the inland "paulis", around Pauli sa Ussa). Sandy soils facilitate drainage of excess water in winter and are suitable for vines and fruit. Finally, the Mogoro river delta records confirm the possible connection of the Punic settlements with the sea through the di Sassu lagoon (mid 1st millennium BC).

\section{Introducción}

Muchos yacimientos arqueológicos costeros mediterráneos de época fenicio-púnica que continuaron activos hasta la época romana o medieval, contienen registros sedimentarios con gran cantidad de datos ambientales, tal y como lo demuestran artículos científicos interdisciplinares y con enfoque geoarqueológico (Marriner y Morhange, 2007; Anthony et al., 2014; Morhange et al., 2015). Por esta razón, en las últimas décadas, la investigación geoarqueológica ha incrementado la información sobre los cambios geomorfológicos y el paleoambiente holoceno e histórico en muchos sectores de la costa mediterránea, en especial en deltas y sistemas de playa barrera-lagoon.

En el entorno de la población de Terralba, situada en el suroeste de la costa mediterránea de la Isla de Cerdeña (Italia) (Fig. 1), se llevaron a cabo trabajos de prospección arqueológica extensiva. Estas prospecciones detectaron una gran concentración de asentamientos rurales (131) de época púnica y romana que se articulaban en torno a Santa Maria di Neapolis, asentamiento seguramente portuario, fundado en el siglo VI a.C. a orillas de un amplio lagoon (Van Dommelen, 1998; 2003). Desde el año 2003, con el fin de documentar las actividades productivas y el paleopaisaje de los asentamientos púnicos, se vienen realizando estudios interdisciplinares cuyos resultados confirman que se trata de establecimientos rurales datados entre los siglos I y V a.C. (Van Dommelen et al., 2006; Van Dommelen et al., 2012; Van Dommelen, et al., in press). En una de las fases del proyecto (2007) se realizó la excavación de uno de estos yacimientos denominado Truncu 'e Molas. Allí se hallaron evidencias de producción de vino y material paleobiológico de la vegetación local y de la ganadería y la dieta de los habitantes de la granja (Pérez Jordà et al., 2010; Van Dommelen, et al., in press). Entre los años 2009 y 2011, con el propósito de evaluar la capacidad agrícola de los suelos del entorno de los yacimientos, se realizó un extensivo estudio geomorfológico y edafológico en los alrededores de otra granja, en la zona denominada Pauli Stincus. Como resultado de estas investigaciones se concluyó que las granjas púnicas se ubicaban predominantemente sobre pequeñas alineaciones arenosas donde las condiciones del suelo eran muy adecuadas para el crecimiento de cultivos arbustivos (diversos tipos de frutales y olivos) (Nicosia et al., 2013).

La investigación arqueológica enfocada al estudio de modelos de ocupación rural en la antigüedad, aporta datos acerca de la actividad productiva de los grupos humanos y también de las pautas de la distribución espacial y temporal de los yacimientos. Este enfoque presenta un gran potencial de investigación desde la perspectiva geoarqueológica. En primer lugar porque facilita la interpretación de las múltiples y variadas interrelaciones existentes entre la acción humana y el medio natural $y$, en segundo lugar, porque aporta gran cantidad de información acerca del cambio ambiental relacionado con la acción antrópica. Ambos temas son de gran interés en los estudios de paisajes rurales históricos y también en los estudios de cambio global. Desde esta perspectiva los objetivos de nuestro estudio son, por un lado, caracterizar las variables del medio físico de los yacimientos de época púnica y, por otro, analizar los posibles cambios ambientales acaecidos en su entorno a través del tiempo. Para ello nos basamos en un estudio geomorfológico de detalle y en el análisis de varios registros sedimentarios en diferentes ambientes.

\section{Materiales y métodos}

La metodología se ha basado en el análisis geomorfológico, estratigráfico y sedimentológico. Se ha integrado además información de diversas fuentes cartográficas, datos históricos y geoarqueológicos. Durante los trabajos de campo de septiembre de 2009 y julio de 2010 y 2014 se reconocieron las diferentes unidades geomorfológicas, se describió la estratigrafía de diversos cortes naturales y artificiales así como 


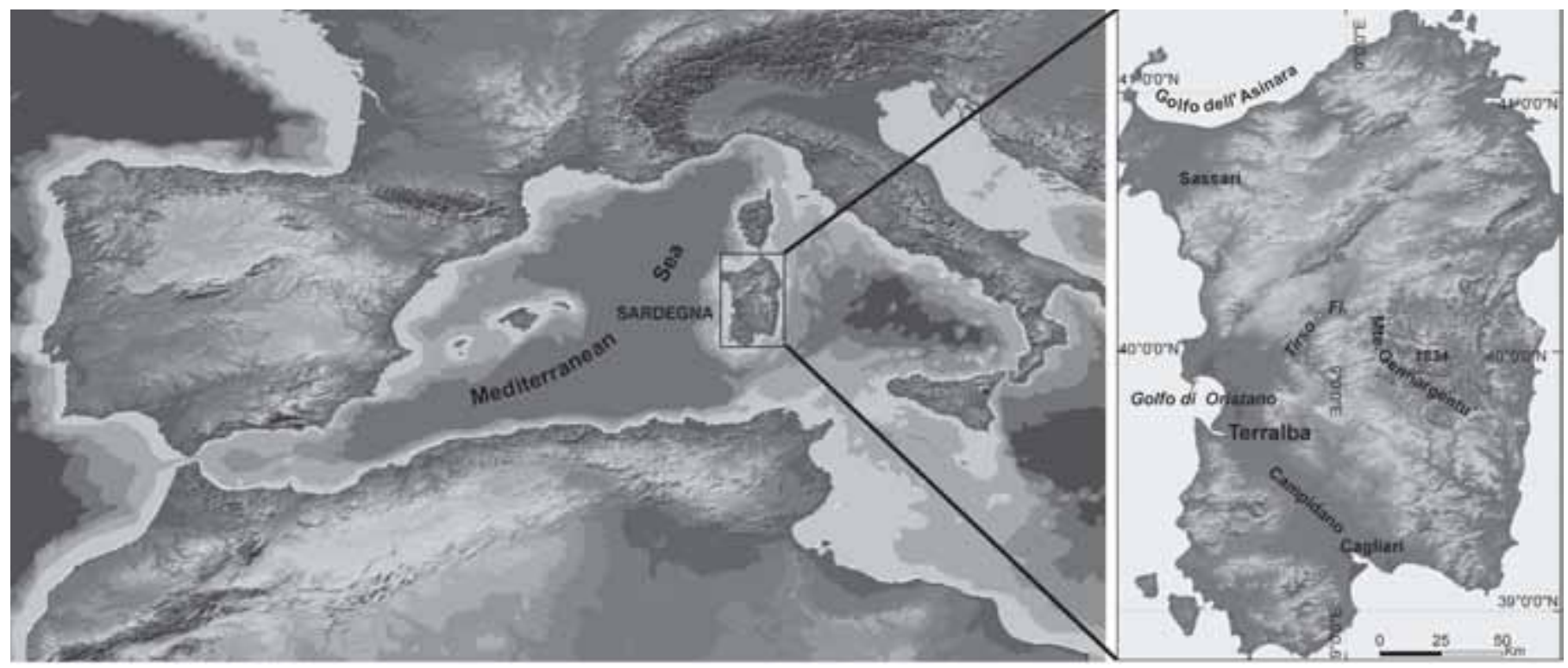

Figura 1. El mar Mediterráneo y la Isla de Cerdeña. Situación del área de estudio.

Figure 1. Mediterranean Sea and island of Sardinia. Situation of the study area.

de la excavación arqueológica de Pauli Stincus. Por otro lado, se realizaron varios sondeos manuales con sonda Eijkelkamp (se alcanzaron profundidades entre 3 y $4.5 \mathrm{~m}$ ) en diversos ambientes como el delta del Mogoro, humedales en torno al Pauli Sa Ussa y en las marismas de Neapolis. Se realizaron tres dataciones de $14 \mathrm{C}$.

Por otro lado, se recopilaron mapas históricos procedentes del archivo de la "Società Bonifiche Sarde" de Arborea, en particular la serie de planos del "Istituto Geografico Militare" del año 1900 a escala 1:25000 y la "Planimetría della Tenuta della Società Bonifiche Sarde di Terralba" a escala 1:10000. Estos planos permiten reconocer rasgos geomorfológicos generales y también cambios recientes asociados a las intensas transformaciones realizadas para la desecación del lagoon de Sassu, la puesta en explotación de la llanura de Arborea y los desvíos y canalizaciones de los ríos Mannu y Mogoro, entre otros.

Los datos geográficos procedentes de las diversas fuentes de información se digitalizaron y trataron con ArcGIS y Microstation V8. Entre las fuentes cartográficas actuales (disponibles en el sistema de descargas oficial de la cartografía de la Regione Autonoma della Sardegna, http://www.sardegnageoportale.it/), se han utilizado mapas ráster escala 1:25000, planos vectoriales 1:10000 (con equidistancia de curvas $10 \mathrm{~m}$ ), ortofotografías aéreas desde 1954 y mapas geológicos entre otros. Cabe resaltar el empleo de un modelo de elevaciones Lidar de alta resolución $(1 \mathrm{~m})$ de buena parte de la Ilanura del Golfo de Oristano, si bien no están disponibles algunas de las zonas con yacimientos en Terralba. Para el resto del área se dispone del MDT de $10 \mathrm{~m}$ de resolución.

\section{Marco regional del área de estudio}

El área de estudio abarca una extensa zona rural de la Ilanura costera en torno a Terralba, localidad ubicada al norte de la depresión del Campidano, próxima al Golfo de Oristano, en la costa occidental de Cerdeña (Fig. 2). Terralba se ubica a $9 \mathrm{~m}$ s.n.m. y a una distancia de unos $10.5 \mathrm{~km}$ de la costa. La llanura está enmarcada por diversos relieves relativamente pronunciados como son el Monte Arci $(812 \mathrm{~m})$ por el E, el Monte Arcuentu $(784 \mathrm{~m})$ y el Monte Linnas (1236 m) por el S. Dos promontorios rocosos, el Capo della Frasca por el S y el Capo San Marco (Tharros) por el $\mathrm{N}$, confieren una morfología ovalada al Golfo de Oristano cuyos fondos son arenosos y muy someros. La llanura se prolonga hacia el interior por el SE a través del amplio corredor del Campidano que utilizan las vías de comunicación hacia el $\mathrm{S}$ de la isla y su capital Cagliari. Numerosos lagoons (denominados Stagno en los mapas topográficos oficiales), algunos desecados durante el siglo $\mathrm{XX}$, aparecen en la línea de costa y se extienden hacia el interior. Además, existen muchas pequeñas áreas palustres (denominadas Pauli en los mapas topográficos oficiales), la mayoría de las cuales están en la actualidad bonificadas o colmatadas. El ríoTirso, el mayor y más cauda- 
loso de la isla, desemboca a la altura de la ciudad de Oristano. De aquí se derivan caudales hacia el canal Tirso-Arborea que irriga en la actualidad buena parte de la llanura. La franja más cercana a la costa, en torno a la población de Arborea, fue objeto de una extensa transformación agraria, con una parcelación rectangular en la década de 1930. Fue entonces cuando se desecó ("Bonifiche Sarde") el lagoon de Sassu, el de mayores dimensiones, situado a unos $4.7 \mathrm{~km}$ al NO de Terralba, para la puesta en cultivo de sus tierras. Estas transformaciones agrarias han modificado total y sustancialmente la morfología natural de los ambientes previos de toda la franja costera.

Desde el punto de vista estructural, Cerdeña es un fragmento de corteza continental deformada por la compresión alpina. Durante el Mioceno finalPlioceno-Cuaternario el régimen extensivo, relacionado con la apertura de la cuenca tirrénica, induce la formación del rift sardo, de orientación aproximadamente N-S (desde el golfo de Cagliari al Golfo de Asinara), sobreimpuesto en su porción suroeste por el graben del Campidano (Cherchi y Montadert, 1982; Pala et al., 1982; Casula et al., 2001). La cuenca del Campidano es un semigraben limitado por una falla principal al este del cabo de San Marco (Fais et al., 1996), se extiende aproximadamente en dirección

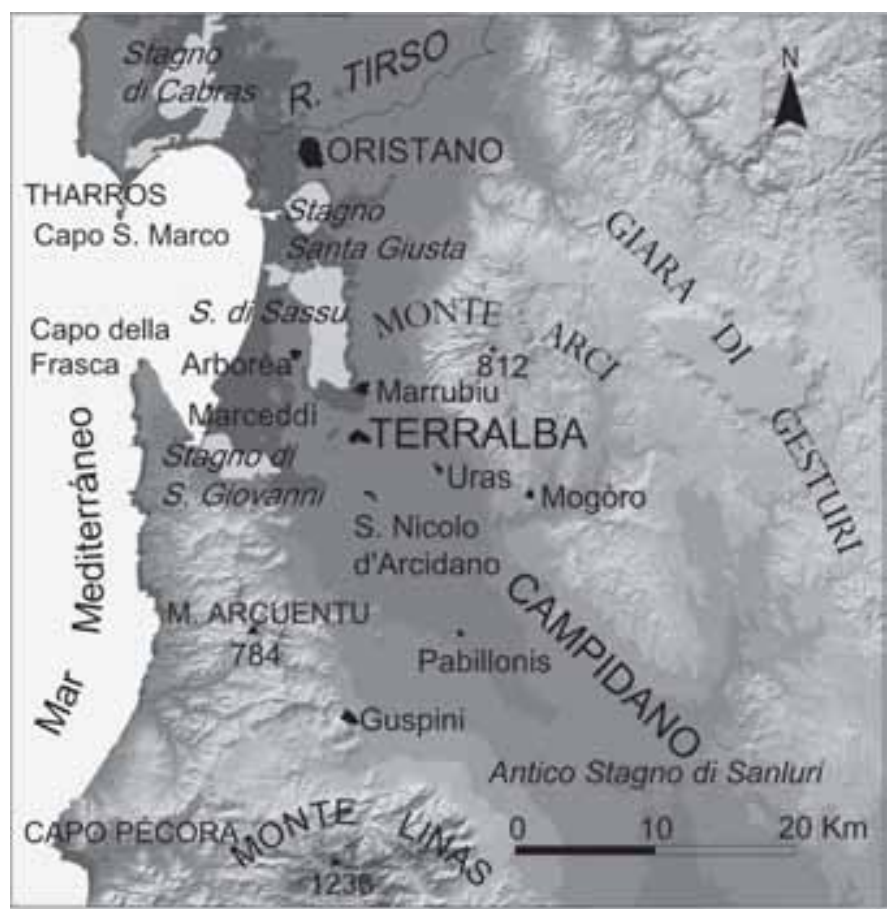

Figura 2. Relieve del Campidano y el golfo de Oristano. Figure 2. Relief of the Campidano and the Oristano Gulf.
NO-SE y está bordeada por dos horst paleozoicos granítico-metamórficos (Fig. 3). Durante el Mioceno se produjeron diversas fases de volcanismo andesítico, probablemente ligado a la zona de subducción, relacionadas con la apertura de la cuenca AlgueroProvenzal. Sedimentos terciarios recubren diferentes tramos del graben del Campidano, como consecuencia de varias transgresiones marinas. Un nuevo ciclo de subsidencia se produjo durante el Plioceno medio, con rellenos lacustres y fluviales hacia el sur y actividad volcánica en su extremo septentrional (graben de Oristano). La composición cambió de ácida (riolitas del Monte Arci) a alcalina (Beccaluva et al., 1977). En la actualidad no hay actividad volcánica y hay una relativa calma sísmica (falta de terremotos históricos y sismicidad registrada instrumentalmente) (Angelone et al., 2005).

Las fases marinas regresivas y transgresivas cuaternarias dejaron en la costa occidental de Cerdeña una alternancia de depósitos marinos, continentales y de transición. Las unidades geocronológicas y litológicas cartografiadas en los mapas geológicos disponibles a escala 1:25000 (http://www.sardegnageoportale.it/) se componen de una extensa banda de materiales cuaternarios (lagunares, marinos, eólicos, fluviales y de piedemonte) que se extiende a lo largo de la cuenca del Campidano y el Golfo de Oristano. EI límite entre las facies marinas y continentales se sitúa en torno al Pauli Sa Ussa al oeste de Terralba (Fig. 4). Dentro del área estudiada, se dató un nivel marino a -14 m recuperado en un sondeo en el Stagno di Sassu con una fecha mayor a 300 ka B.P. (Pretirreniense antiguo), correspondiente al estadio isotópico marino 9c (Crotoniense) (Belluomini and Delitala, 1988; Spano et al., 2005). Son frecuentes, asimismo, los afloramientos tirrenienses (120-100 ka) situados entre 2-4 m s.n.m. en numerosos puntos de la costa sarda y correspondientes a un interglacial más cálido que el actual. Un caso bien estudiado se encuentra en la península de S. Giovanni di Sinis (al norte del Golfo de Oristano, cerca de Tharros) (Fig. 4) (Belluomini et al., 1986; Lecca and Carboni, 2007; Andreucci et al., 2009). Durante el estadio MIS 5.5 se depositaron sedimentos marinos (conglomerados y arenas), de playa emergida y sumergida, con fauna fósil subtropical de afinidad senegalesa (Strombus bubonius y Patella ferruginea) que permiten referirlos a la transgresión Tirreniense, hacia 125 ka B.P. Por encima aparece un potente nivel eólico post-Tirreniense. Los depósitos continentales se caracterizan principalmente por arenas eólicas y aluviales que se atribuyen a la última regresión marina pleistocena (Würmiense) (Carmignani et al., 2001; Andreucci et al., 2010).

Por lo que respecta al clima, la isla de Cerdeña 


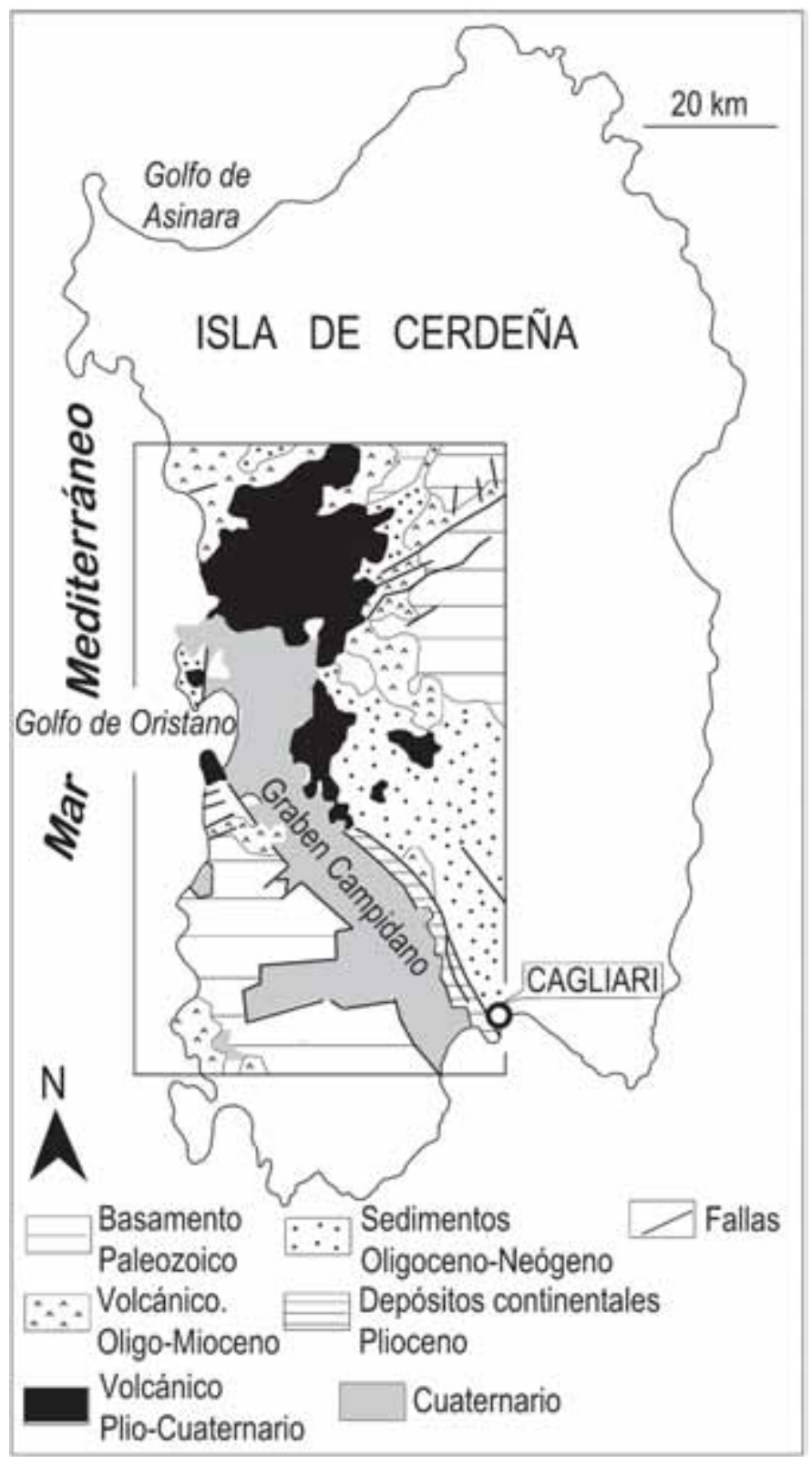

Figura 3. Geología del Campidano y el Golfo de Oristano (modificado de Casula et al., 2001).

Figure 3. Geology of the Campidano and the Oristano Gulf (modified from Casula et al., 2001).

muestra una gran variabilidad espacial debido a los efectos del relieve (la altitud, la orientación de la costa y la distancia al mar). En la llanura de Terralba los registros de precipitaciones anuales rondan los 600-650 mm (Ente Autonomo del Flumendosa, 1998). En las montañas próximas se superan los $1000 \mathrm{~mm}$ en el Monte Arci y los $1200 \mathrm{~mm}$ en el Monte Linas. La variabilidad interanual no es demasiado acusada, en Arborea el año más lluvioso (1979) supera los 1000 $\mathrm{mm}$ y el más seco (1983) apenas los $400 \mathrm{~mm}$. El verano es muy seco (entre junio y agosto sólo se registran $22 \mathrm{~mm}$ ) y el período lluvioso se concentra en otoño e invierno. Las temperaturas medias anuales rondan los $16.5-17^{\circ} \mathrm{C}$ en la llanura costera. Otros factores climáticos importantes son el régimen de vientos y brisas y la nubosidad que tienden a incrementar la humedad ambiental. La disposición del relieve y la orientación de la costa dejan el golfo de Oristano y la Ilanura de Terralba muy expuesta al Maestrale (viento del noroeste). La descarga hidrológica de los principales cauces del Campidano septentrional cercanos a Terralba se concentra en el otoño-invierno con crecidas súbitas que suelen producir inundaciones. Durante el largo estiaje en verano los cauces quedan prácticamente en seco. El caudal medio mensual de febrero alcanza $4.69 \mathrm{~m}^{3} / \mathrm{seg}$ en el Mannu, $3.66 \mathrm{~m}^{3} / \mathrm{seg}$ en el Mogoro y $1.48 \mathrm{~m}^{3} / \mathrm{seg}$ en el Sitzerri.

\section{Rasgos geomorfológicos}

Los asentamientos rurales de época púnica y romana se ubican sobre la amplia Ilanura aluvial en torno a la población de Terralba, entre los llanos de inundación de los ríos Sitzerri-Mannu y Mogoro. La llanura está modelada por mantos arenosos y humedales de diverso tipo. El trabajo de campo, la interpretación de fotografías aéreas y el análisis de la topografía en el modelo de elevaciones del terreno nos han permitido reconstruir los principales rasgos y procesos geomorfológicos de la zona (Fig. 4).

\section{La morfología de la costa}

La costa del golfo de Oristano es de ambiente micromareal, con un rango de $0.2 \mathrm{~m}$ (en Cagliari), que sólo es excedido por las fluctuaciones del nivel del mar relacionadas con cambios en la presión barométrica o con los efectos del viento. Está formada por una playa arenosa, regularizada por la corriente de deriva litoral (N-S) que redistribuye sedimentos desde la desembocadura del río Tirso y desde la somera plataforma marina adyacente. Junto a la playa se adosa un cordón con pequeñas dunas. Varias flechas litorales de morfología ligeramente curvada que parten del delta de oleaje del ríoTirso se interrumpen en la bocana artificializada del lagoon de S'Ena Arrubia y se prolongan hacia el sur hasta la punta Corru Mannu (Figs. 4 y 5). En conjunto se trata de una costa de cordones arenosos progradantes y flechas (Fig. 5) que parten del delta de oleaje del río Tirso (Fig. 4). Estos cordones conforman cierres que aíslan del mar lagoons de 


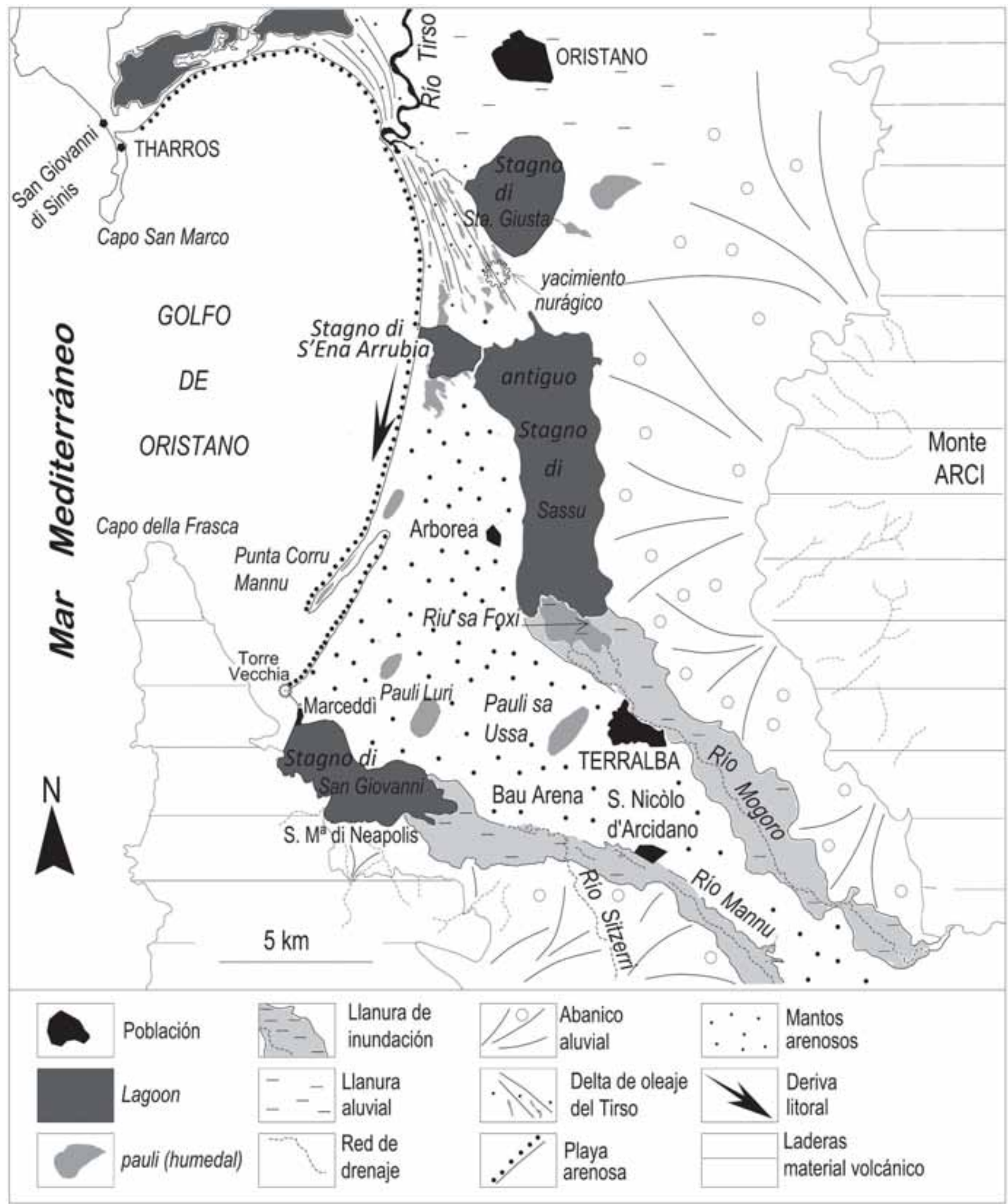

Figura 4. Mapa geomorfológico del entorno de Terralba y del Golfo de Oristano.

Figure 4. Geomorphologic map around Terralba and the Oristano Gulf. 
morfología alargada, tales como el Stagno (Peschiera) de Corru s'Ittiri, el Pauli Pirastu y el Pauli Ghilemu que se comunican con lagoons cercanos al Stagno de s'Ena Arrubia (Fig. 5). Una extensa rasa marina pleistocena aflora en el extremo costero meridional de la plataforma, en el área de Marceddì (mapa geológico 1:25000 en http://www.sardegnageoportale.it/). La rasa se extiende centenares de metros con varios decímetros de profundidad en la playa contigua.

\section{Los sistemas fluviales}

Hacia el sector meridional y oriental de Terralba la lla-

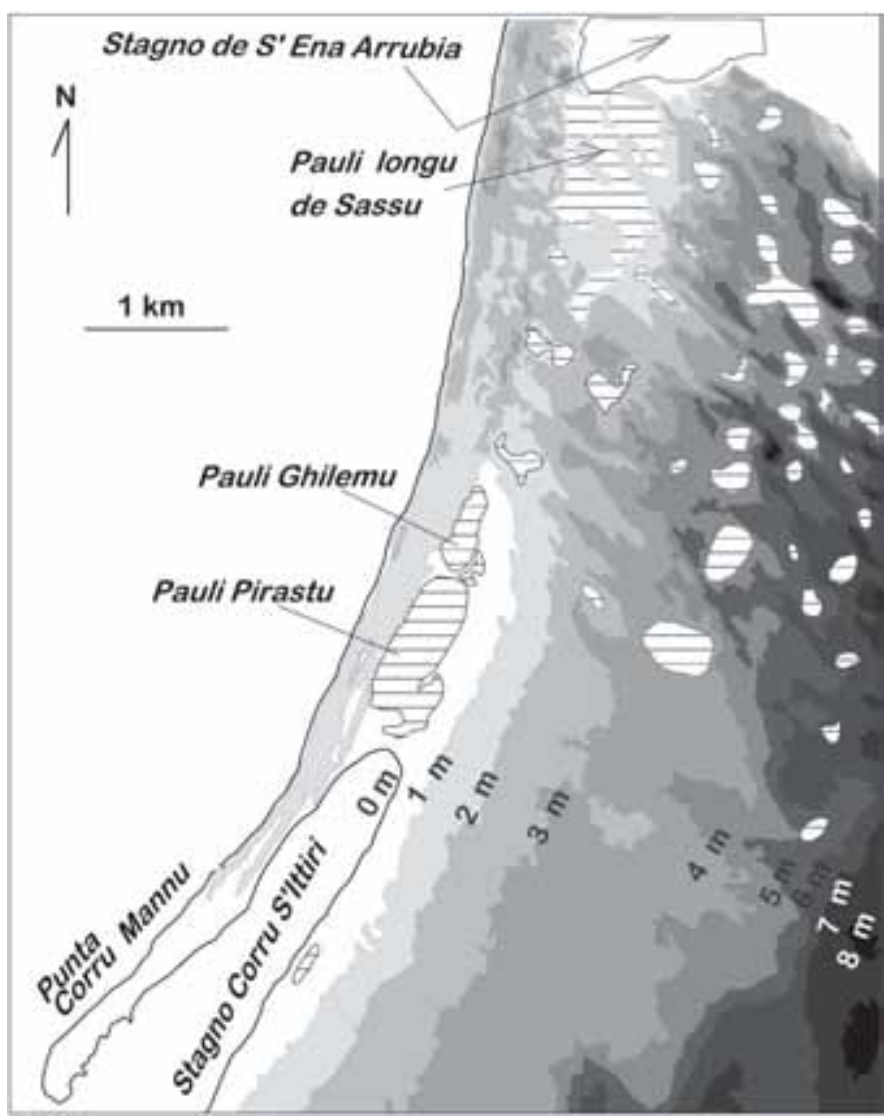

Figura 5. Rasgos morfológicos del litoral del golfo de Oristano. Las áreas con rayado horizontal representan humedales. La topografía entre 3 a $8 \mathrm{~m}$ s.n.m. se corresponde con alineaciones dunares (reelaborado a partir de la Planimetria della Tenuta della Societa Bonifiche Sarde di Terralba. Escala original 1:10000; proporcionada amablemente por el Sr. Manca).

Figure 5. Morphological patterns of the littoral of the Oristano Gulf. Areas with horizontal striping represent wetlands. The topography between 3-8 $m$ above sea level corresponds to dune alignments (redrawn from the Planimetria della Societa della Tenuta di della Societa Bonifiche Sarde di Terralba. Original Scale 1: 10000; kindly provided by Mr. Manca). nura está limitada por las acumulaciones fluviales y de piedemonte del monte Arcuentu-Linas y del Monte Arci. Los cauces fluviales que descienden de los pronunciados relieves paleozoicos de rocas volcánicas, filonianas, graníticas y metamórficas del monte Arcuentu-Linas, dejan extensos depósitos de gravas y arenas con morfología de abanicos aluviales de fuerte pendiente. En numerosos tramos se han realizado canalizaciones para evitar las migraciones laterales y desapariciones de los cauces naturales. Desde las vertientes orientales descienden pequeños barrancos del Monte Arci que desaparecen sobre los piedemontes o bien conforman pequeños abanicos en el borde del antiguo lagoon di Sassu (Fig. 4).

Además de estos sistemas fluviales menores, en el entorno próximo a Terralba se localizan dos valles fluviales principales, por el $\mathrm{S}$, el valle del río Mannu que discurre junto a San Nicolò d'Arcidano y, por el O, el valle del río Mogoro cuyo llano de inundación discurre junto a la población de Terralba. La cuenca de drenaje (unos $351 \mathrm{~km}^{2}$ ) del río Mogoro abarca las vertientes occidentales y meridionales del Monte Arci (803 m). Antes de su desvío y encauzamiento, se bifurcaba en varios canales que desembocaban en el Stagno de Sassu, tal como representa el mapa del año 1900 del Istituto Geografico Militare, 1:25000 (Riu sa Foxi en Fig. 4). La cuenca de drenaje conjunta de los ríos Mannu-Sitzerri $\left(599 \mathrm{~km}^{2}\right)$ tiene sus principales aportes de sedimentos en las cabeceras orientales de los relieves graníticos y metamórficos del monte Linas $(1236 \mathrm{~m})$ y en los relieves basálticos del Monte Arcuentu $(784 \mathrm{~m})$. El río Mannu desciende canalizado desde su cabecera en la depresión del Campidano hasta su desembocadura en el lagoon de Santa Maria di Neapolis. Por otro lado, antes de ser encauzado, el río Mannu discurría con varios canales someros (sin orillas) por un valle encajado entre mantos arenosos eólicos hacia el norte y abanicos aluviales hacia el sur. Tras su confluencia con el torrente Sitzerri, a la altura del Bau Arena, su curso describía algunos meandros de pequeña amplitud antes de desembocar en el lagoon de Santa Maria (orilla del yacimiento arqueológico homónimo) donde forma un delta interior.

El fondo de los valles de ambos ríos (Mannu y Mogoro) está recubierto de limos y arcillas de inundación que conforman una extensa y lisa llanura de inundación de varios centenares de metros de anchura. En los laterales del valle pueden reconocerse puntualmente depósitos de gravas fluviales que corresponden a terrazas pleistocenas (mapa geológico 1:25000 en http://www.sardegnageoportale.it/). Salvo el río Tirso, el único con desembocadura marina, los cauces principales afluentes a la llanura desembocan 
de manera natural en lagoons donde forman deltas interiores (Fig. 4).

\section{Los sistemas eólicos}

Materiales cuaternarios marinos, continentales y de transición conforman la plataforma en la que se asientan los yacimientos en estudio, en torno a las poblaciones de Arborea, Terralba y San Nicolò d'Arcidano (Fig. 4). Se trata de una plataforma pleistocena-holocena recubierta por extensos mantos arenosos (mapa geológico 1:25000 en http://www.sardegnageoportale.it/) que localmente pueden formar

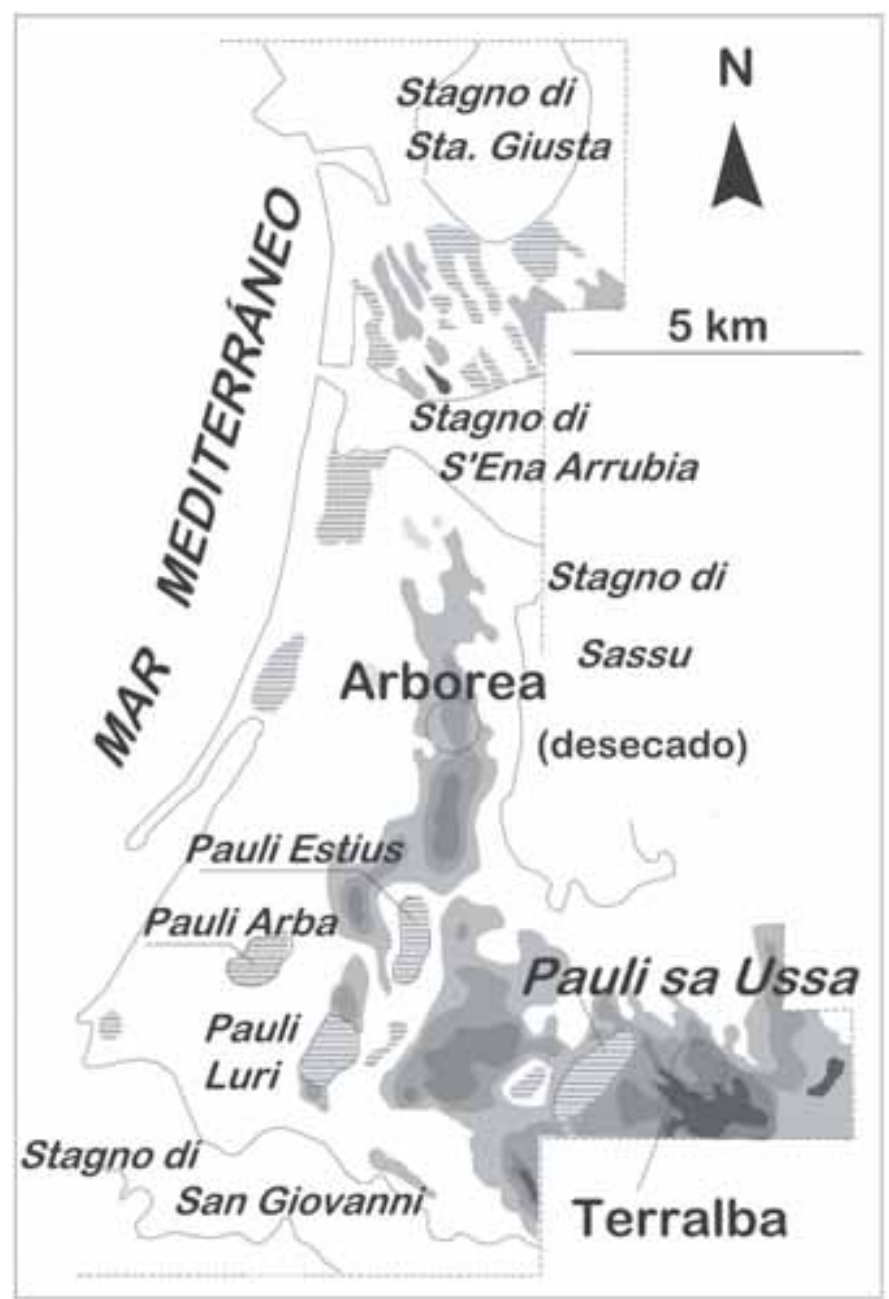

Figura 6. Disposición general de los mantos arenosos o cordones dunares según cartografía LIDAR. Las áreas con rayado horizontal representan "paulis".

Figure 6. General arrangement of dune ridges as LIDAR mapping. Areas with horizontal striping represent "paulis"(wetlands). cordones (elevaciones de morfología alargada) de entre 8 y $15 \mathrm{~m}$ s.n.m. Es importante destacar que aunque la morfología dunar original de toda la franja costera arenosa fue totalmente transformada durante la "Bonifiche" de Mussolini (Soru, 2000), la cartografía Lidar con resolución de $1 \mathrm{~m}$ todavía permite reconocer algunos rasgos morfológicos de las dunas (Fig. 6).

Los extensos mantos arenosos recientes son el elemento más característico de la geomorfología del área. Su peculiar y compleja morfología puede estar relacionada con la dinámica eólica específica, con la existencia de afloramientos pleistocenos subyacentes $y$, en ocasiones, con la neotectónica y/o subsidencia diferencial a lo largo de la llanura costera. Los mantos eólicos costeros se alimentan continuamente desde las playas por vientos del NO (Maestrale), conformando incipientes cordones dunares. No obstante, las arenas penetran hacia el interior varios kilómetros y alcanzan la zonas de Terralba y San Nicolò d'Arcidano (Fig. 7), donde recubren unidades aluviales y relieves residuales de época cuaternaria y neógena. Cerca de Arborea la altura de los mantos arenosos o antiguas dunas alcanza entre 5 y $8 \mathrm{~m}$ de altura y conforman una extensa acumulación arenosa de trazado paralelo a la costa. Tierra adentro, hacia Terralba, la altura de los mantos eólicos alcanza entre 10 y 16 m s.n.m., mientras que en la franja costera habitualmente no sobrepasan los 2-4 $\mathrm{m}$ de altura (Figs. 5 y 6). En las acumulaciones arenosas se detectan dos direcciones predominantes, una de ellas (NOSE) está orientada por los vientos que recorren la fosa del Campidano y están ligadas genéticamente al paso de borrascas invernales. Otras alineaciones se disponen paralelas al litoral y se asocian a vientos costeros. No obstante, tal y como hemos remarcado, en la actualidad la morfología de las antiguas dunas costeras está muy transformada por los cultivos y en especial por el parcelario cuadrangular implantado durante la bonificación (Fig. 7).

\section{Los ambientes de lagoon y palustres}

La llanura costera está formada por extensas lagoons litorales tales como los stagnos de Marceddì, de San Giovanni, de Sassu, de s'Enna Arrubia, de Corru s'Ittiri y Santa Giusta (Figs. 4 y 5). Estos lagoons son posiblemente los ambientes residuales del extenso estuario originado durante la transgresión marina holocena. En la actualidad se trata de lagoons con diferentes grados de salinidad (hipersalinas a salobres) según la influencia y apertura marina (Magni et al., 2008). Además de estos extensos lagoons, se distinguen multitud de pequeñas $y$ medianas áreas 


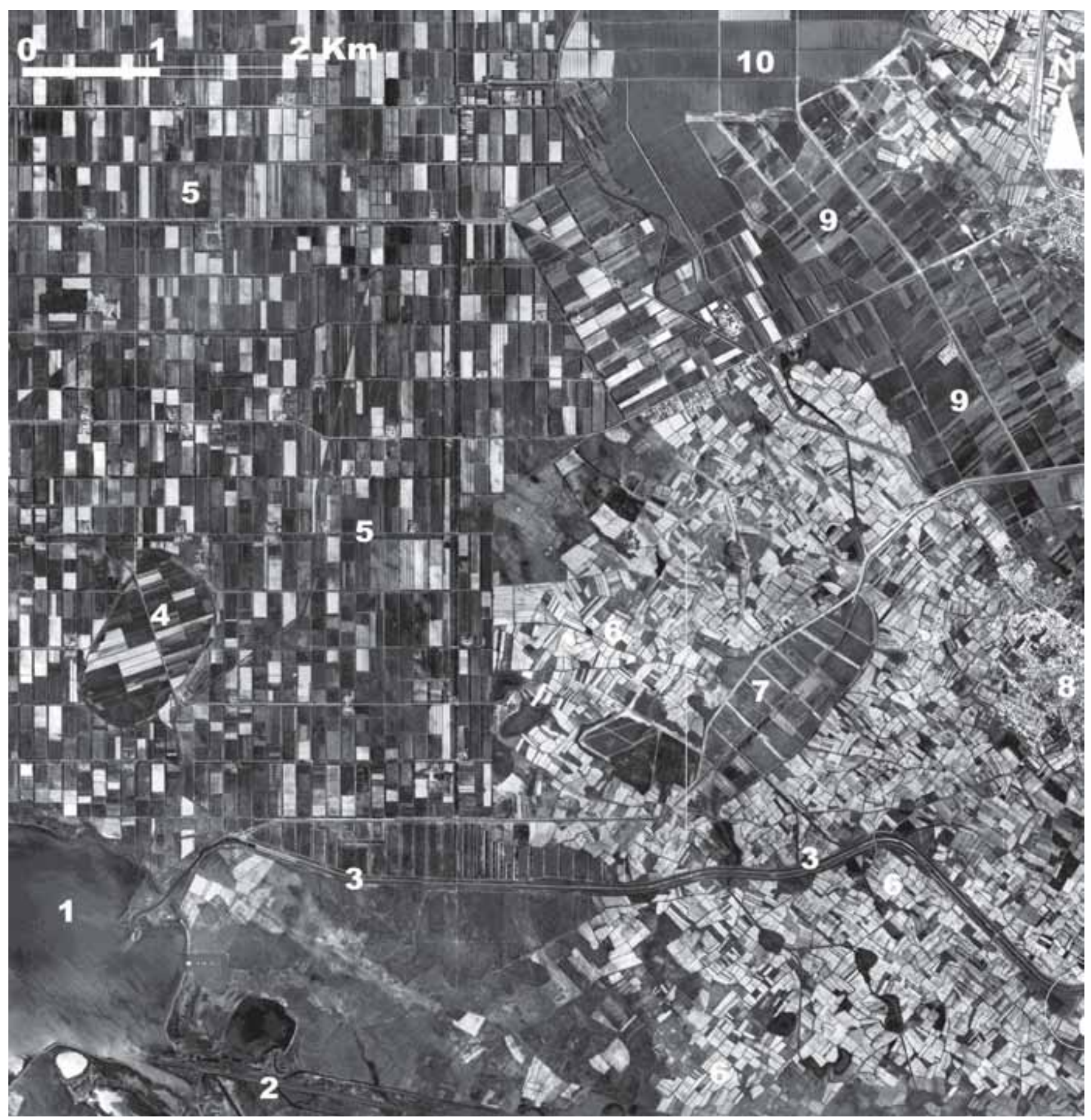

Figura 7. Diferentes ambientes de la llanura de Terralba sobre la fotografía aérea de 1968: 1) Stagno di San Giovanni, 2) Desembocadura artificial del río Mannu-Sitzerri, 3) Cauce desviado del río Mogoro, 4) Pauli Luri, 5) Mantos eólicos transformados en parcelas rectangulares durante la Bonifica de Mussolini al sur de Arborea (sector occidental de la fotografía áerea), 6) Ambientes de dunas y "paulis" de la colonización púnica (parcelas irregulares en el sector sureste de la fotografía aérea), yacimiento Pauli Stincus señalado en un círculo, 7) Pauli sa Ussa, 8) Terralba, 9) Llanura de inundación y llanura deltaica del río Mogoro, 10) Stagno di Sassu (desecado).

Figure 7. Different environments of the Terralba plain on the 1968 aerial photograph: 1) Stagno di San Giovanni, 2) Artificial outlet of the Mannu-Sitzerri river, 3) Diversion channel of Mogoro river, 4) Pauli Luri, 5) Aeolian mantles and "paulis" (wetlands) transformed in rectangular plots by reclamation works during Mussolini's regime (south of Arborea) (western sector of the aerial photograph), 6) Dune and "pauli" (wetland) environments of Punic settlement (irregular plots on the southeastern sector of the aerial photograph), archaeological site of Pauli Stincus marked in a circle, 7) Pauli sa Ussa, 8) Terralba, 9) floodplain and deltaic plain of the Mogoro river, 10) Stagno di Sassu (drained lagoon). 
palustres localmente denominadas "pauli". El topónimo se repite con mucha frecuencia en la cartografía, aunque, como hemos podido comprobar, no siempre tiene el mismo significado morfogenético.

En efecto, existen áreas palustres de morfología, dimensiones y procesos muy variados. Las mayores tales como el Pauli Estius, el Pauli Arba o el Pauli sa Ussa tienen varios centenares de metros de longitud, una morfología de depresión alargada y una disposición, orientación y tamaño similar a las actuales backbarrier lagoons (Figs. 5 y 6). El Pauli Luri, ubicado en una depresión dentro de una extensa acumulación arenosa, debe tener su origen en procesos de subsidencia localizada.

Por otro lado, la disposición de las elevaciones arenosas en series, alargadas decenas de metros, en dirección NO-SE y elevados topográficamente, configura espacios deprimidos (pasillos de deflación entre dunas longitudinales) en los que aflora estacionalmente el nivel freático. Este tipo de humedales de apenas decenas de metros de longitud eran muy frecuentes en la franja costera, tal y como puede observarse en el detallado levantamiento topográfico previo a la transformación agraria (Fig. 5), pero todos

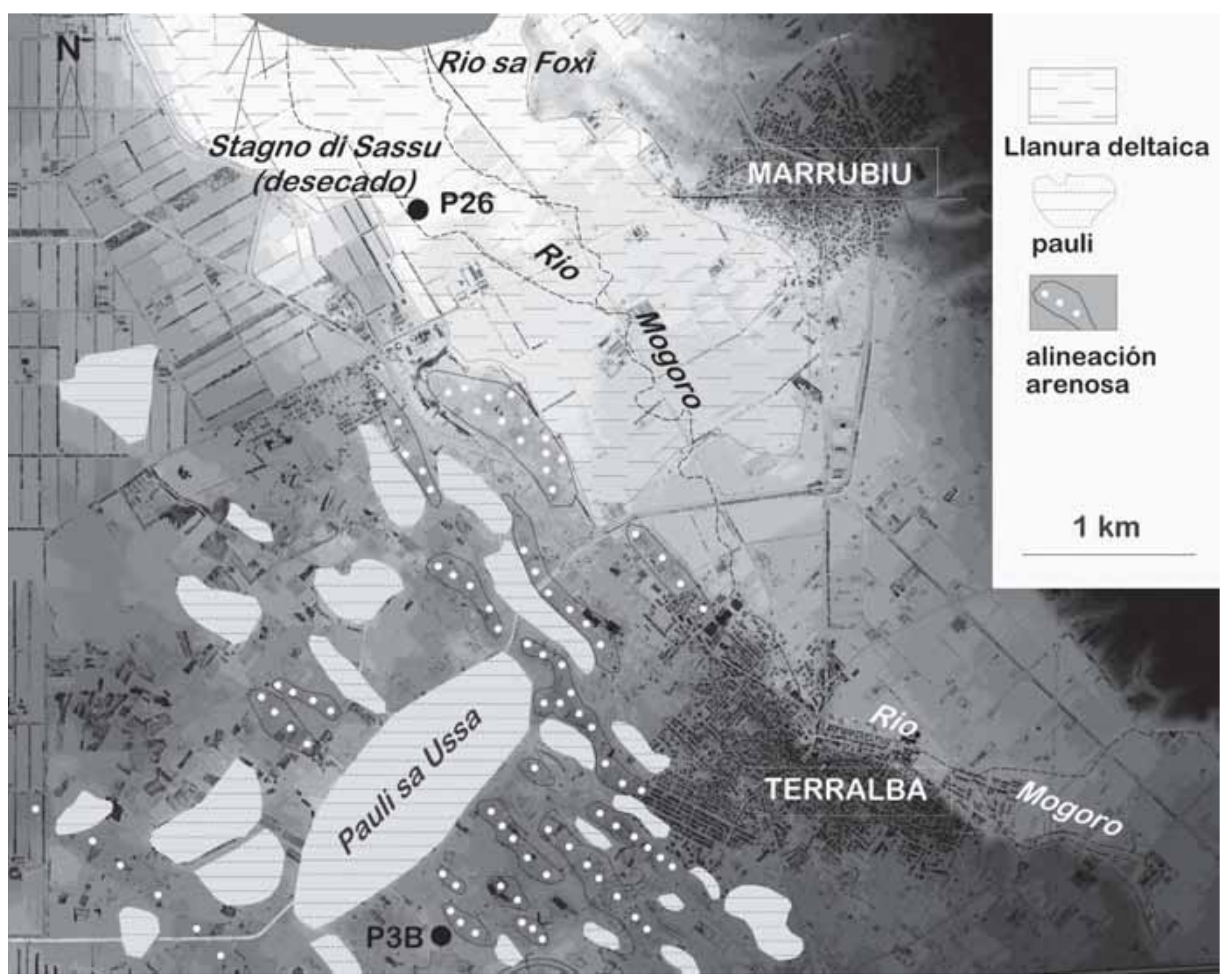

Figura 8. Pauli sa Ussa. Las áreas de deflación eólica quedan representadas con rayas horizontales y las alineaciones arenosas o antiguos cordones dunares con punteado en blanco. El punto negro cerca del lagoon de Sassu y el situado al sur del Pauli sa Ussa indican la ubicación de los registros P26 y P3B (respectivamente).

Figure 8. Pauli sa Ussa. The wind deflation areas are represented horizontally striped and the dunes are dotted in white. The dark spot near the lagoon of Sassu and south of the Pauli sa Ussa indicates the location of the P26 and P3B records (respectively). 
fueron totalmente arrasados y transformados durante la bonificación ("Bonifiche sarda"). Finalmente, existe otro tipo de "paulis" o humedales en posición más continental que son muy abundantes en el entorno deTerralba y el Pauli sa Ussa (Figs. 7 y 8). Este tipo de humedales se ubica igualmente entre estrechas $y$ delgadas alineaciones arenosas o cordones dunares (con la misma dirección NO-SE que los mantos arenosos costeros de la figura 5) y, en los trabajos de campo, hemos comprobado que se corresponden con áreas de deflación eólica en las que aflora el sustrato de arcillas montmorilloníticas (Ilanura fluvial pleistocena). La topografía del fondo de estos humedales también denominados "pauli" presenta cierto microrrelieve (la base no es plana) y conforma ámbitos muy poco deprimidos desde la perspectiva topográfica. Hoy en día estas depresiones están cultivadas con cereal y el afloramiento estacional de agua en ellas se justifica por el sustrato de arcillas y la alta permeabilidad de las arenas del entorno. Cabe resaltar que esta tipología mixta de "alineación alargada arenosa" y "pauli" es el contexto más habitual de los yacimientos púnicos en el entorno deTerralba (Fig. 8).

\section{Los registros del cambio ambiental de los últimos milenios}

El territorio de mayor concentración de yacimientos púnicos se encuentra en los arenales y humedales en torno a la población deTerralba y el Pauli Sa Ussa, entre las Ilanuras aluviales-deltaicas de los ríos Mogoro y Mannu-Sitzerri. Pasamos a describir los registros sedimentarios analizados en dichos ambientes.

\section{El entorno del Pauli sa Ussa}

Durante los trabajos de campo se reconoció un corte sedimentológico (con cerámicas de época púnica) en una zanja artificial cercana al Pauli sa Ussa (P3B en Fig. 8). La secuencia de 2 metros de potencia se compone de tres niveles diferenciables por la textura, el color y el contenido en materia orgánica (Fig. 9). La base (a), hacia $2 \mathrm{~m}$ de profundidad está formada por un nivel de limos y arcillas muy endurecidos, de color gris verdoso, con grietas de retracción, abundantes rasgos de oxidación, precipitados de manganeso y estructura en pequeños poliedros $(\mathrm{cm})$. En contacto neto se dispone un nivel (b) de arenas $(1.4 \mathrm{~m}$ de potencia) en el que se diferencian: primero $40 \mathrm{~cm}$ de arenas masivas de color verdoso, $50-60 \mathrm{~cm}$ hacia la parte central del corte, que cambia gradualmente a tonos grisáceos $\mathrm{y}$, por encima, en contacto nítido, 30-
$40 \mathrm{~cm}$ de arenas de color gris oscuro, con abundantes restos de plantas carbonizadas, gasterópodos Lymnaea palustris, restos vegetales y fragmentos cerámicos de época púnica. Se dató una concha de gasterópodo mediante 14C AMS (490 a 460 años cal B.C. / 420 a 390 años cal B.C.) (Tabla 1), fecha que concuerda con los fragmentos cerámicos de época púnica que aparecen en este sector del corte. El nivel superior (c) (de potencia muy variable, entre 60-50 $\mathrm{cm}$ ) se compone de una capa de arenas muy sueltas de tono gris-marrón con ligera proporción de limo y con abundantes raíces y gasterópodos terrestres. Este nivel contiene fragmentos cerámicos arqueológicos de diversas épocas.

La base de la secuencia (a) se interpreta como un ambiente palustre asociado al Pauli sa Ussa. El nivel de arenas (b) con alto contenido en materia orgánica se correspondería con un ambiente eólico de época púnica próximo a un humedal de agua dulce (con vegetación y gasterópodos dulceacuícolas). Las arenas sueltas del techo (c) de la unidad sellan este ámbito de humedal de época púnica.

\section{El registro del delta interior del río Mogoro}

Se realizó un sondeo (P26) con sonda Eijkelkamp en la llanura deltaica del río Mogoro a $1.3 \mathrm{~m}$ s.n.m., cauce que antes de su desvío artificial hacia el lagoon de San Giovanni desembocaba en el lagoon de Sassu al norte de Terralba (Figs. 4, 7 y 8 ). Se recuperaron muestras semiperturbadas hasta profundidades de 4 a $5 \mathrm{~m}$, si bien los dos metros superiores se desecharon ya que se trataba de material de rellenos realizados durante la colmatación artificial del lagoon (Fig. 10). El dato más importante a destacar es que, hacia la base, entre 3 y $4 \mathrm{~m}$ de profundidad se alcanzó un nivel de lagoon salobre con Cerastoderma sp. y Abra alba que se dató mediante $14 \mathrm{C}$ (AMS) hacia 800-760 años cal B.C. / 680-670 años cal B.C. a $3.5 \mathrm{~m}$ de profundidad y 1010-890 años cal B.C. / 870-850 años cal B.C. a $4 \mathrm{~m}$ de profundidad (Tabla 1). La textura del nivel de lagoon salobre en la base es de fangos arenosos con alto contenido en materia orgánica. Por encima, entre 2 y $3 \mathrm{~m}$ de profundidad, se superpone un nivel de fangos lagunares con vegetación acuática y Planorbis sp. que se podría interpretar como un tránsito desde el anterior lagoon salobre a un lagoon dulceacuícola.

\section{Registro en el Stagno di S.M. di Neapolis}

Se realizó un sondeo manual en las marismas que 


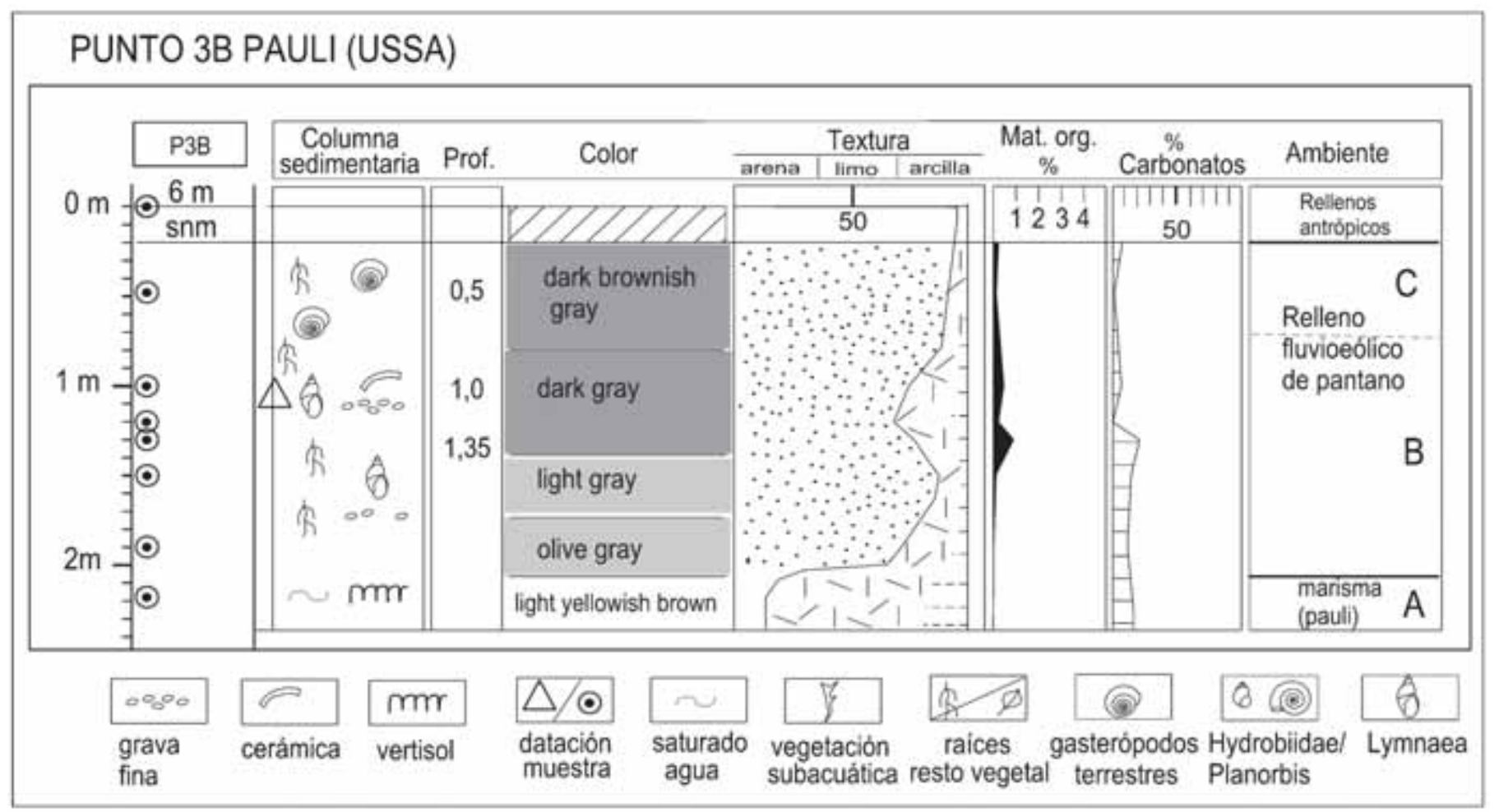

Figura 9. Registro sedimentario de Pauli Sa Ussa (Punto 3B).

Figure 9. Sedimentary record of Pauli Sa Ussa (Punto 3B).

rodean el yacimiento de Santa de María di Neapolis, a 1.7 m s.n.m., en el que se recuperaron muestras semiperturbadas hasta una profundidad de $4.2 \mathrm{~m}$ (Fig. 11). Se distinguen los siguientes niveles: entre 3.25 y $4.2 \mathrm{~m}$ de profundidad aparece un nivel de arenas gruesas sueltas de color gris; por encima, entre 2.3 y $3.25 \mathrm{~m}$ de profundidad, se dispone un nivel de arenas limo arcillosas con alto contenido en materia orgánica y abundantes restos de vegetación acuática; el techo de la secuencia está conformado por un estrato bastante potente ( 0 a $2.3 \mathrm{~m}$ de profundidad) de textura limoarcillosa con ligero contenido en materia orgánica, restos vegetales, abundantes raíces y nódulos dispersos.

Vista las características de la columna sedimentaria cabe pensar que el techo del registro lo componen aportes distales de los cauces afluentes (Mannu y Sitzerri, entre otros) en un ambiente de transición. Por otro lado, puesto que la cota absoluta de las arenas gruesas basales se encuentra por debajo del nivel del mar, cabe preguntarse si su origen corresponde a un ambiente fluviodeltaico o eólico o si bien corresponde a aportes desde el litoral dentro del antiguo estuario de Marceddì-San Giovanni.

\begin{tabular}{|l|l|l|l|l|}
\hline \multicolumn{1}{|c|}{ MUESTRA } & \multicolumn{1}{c|}{ MATERIAL } & \multicolumn{1}{c|}{ FECHA 14C } & \multicolumn{2}{c|}{ EDAD CALIBRADA (2 sigma) } \\
\hline P26-4 $\mathrm{m}$ & $\begin{array}{l}\text { Sedimento } \\
\text { orgánico }\end{array}$ & $2790 \pm 30 \mathrm{BP}$ & $\begin{array}{l}1010-890 \mathrm{cal} \mathrm{BC} \\
870-850 \mathrm{cal} \mathrm{BC}\end{array}$ & $\begin{array}{l}2960-2840 \mathrm{cal} \mathrm{BP} \\
2820-2800 \mathrm{cal} \mathrm{BP}\end{array}$ \\
\hline P26-3.45 m & $\begin{array}{l}\text { Concha } \\
\text { (Cerastoderma sp.) }\end{array}$ & $2570 \pm 30 \mathrm{BP}$ & $\begin{array}{l}800-760 \mathrm{cal} \mathrm{BC} \\
680-670 \mathrm{cal} \mathrm{BC}\end{array}$ & $\begin{array}{l}2750-2710 \mathrm{cal} \mathrm{BP} \\
2630-2620 \mathrm{cal} \mathrm{BP}\end{array}$ \\
\hline P 3B & $\begin{array}{l}\text { Concha } \\
\text { (gasterópodo lagunar) }\end{array}$ & $2360 \pm 30 \mathrm{BP}$ & $\begin{array}{l}490-460 \mathrm{cal} \mathrm{BC} \\
420-390 \mathrm{cal} \mathrm{BC}\end{array}$ & $\begin{array}{l}2440-2410 \mathrm{cal} \mathrm{BP} \\
2370-2340 \mathrm{cal} \mathrm{BP}\end{array}$ \\
\hline
\end{tabular}

Tabla 1. Dataciones ${ }^{14} \mathrm{C}$. Datos utilizados en la calibración de las muestras: INTCAL04 (Reimer et al., 2004).

Table 1. ${ }^{14}$ C Dating. Database used: INTCAL04 (Reimer et al., 2004). 
Ruiz, J.M., et al., 2018. Geomorfología y cambio ambiental en el entorno de los... Boletín Geológico y Minero, 129 (1/2): $331-351$

\section{Discusión}

Teniendo en cuenta los procesos geomorfológicos más relevantes y la información que contienen los registros sedimentarios, se puede plantear una discusión en torno a la caracterización del medio ambiente objeto de la colonización agraria de época púnica y del cambio ambiental histórico en el entorno costero.

\section{Dunas y humedales: el medio ambiente objeto de la colonización agraria de época púnica}

Aunque no disponemos de dataciones absolutas en nuestra área de estudio, cabe destacar la importancia de las unidades eólicas pleistocenas y holocenas en la costa occidental de Cerdeña. Extensivos mantos de arena en la llanura de Arborea-Terralba, ocupan toda la franja costera y penetran varios kilómetros hacia el interior. El gran manto eólico intercalado con capas marinas y aluviales que conforma el sustrato de la llanura de Arborea-Terralba, se formaría durante los episodios de rápido descenso del nivel marino del Pleistoceno superior durante los cuales quedaron expuestas enormes cantidades de arena (Carmignani et al., 2001; Andreucci et al., 2010). La línea de costa se desplazaría entonces hacia el mar dejando expuesta a los procesos eólicos la somera plataforma del Golfo de Oristano (con pendientes muy bajas entre 2.5-4\%o). Cabe destacar el episodio eólico que se produjo en torno a 75 ka B.P. al comienzo del MIS4 (Andreucci et al., 2010), datado en acantilados de la costa occidental sarda. Esta fase es contemporánea a la formación de la unidad $U_{0}$ en El Abalorio en la costa atlántica de Huelva (Zazo et al., 2011), sobre la cual se depositaron posteriormente varias unidades eólicas pleistocenas y holocenas. En Baleares las dunas fósiles o eolianitas risianas (marès) se extienden desde la línea de costa hasta zonas interiores a más de $200 \mathrm{~m}$ por encima del nivel del mar (Henningsen et al., 1981; Rohdenburg, 1992). Dichos autores también atribuyen la mayor parte de estas acumulaciones de eolianitas costeras a fases de regresión marina durante episodios fríos del Würm cuando queda expuesta la plataforma marina.

Por otro lado, la actividad eólica no se limita a la costa y se han detectado aportes de polvo sahariano en paleosuelos pleistocenos del noroeste de Cerdeña, preservándose preferentemente en suelos acumulados y meteorizados durante estadios interglaciares (Andreucci et al., 2012). También, Sevinek y Kummer (1984) señalan el origen eólico del cuarzo y la mica presentes en el relleno de depresiones situadas en los relieves tabulares de la Giara Di Gesturi (al este de Terralba).

En cuanto a las fases eólicas holocenas enTerralba, los únicos datos de los que disponemos son los de yacimientos púnicos como Pauli Stincus (Nicosia et al., 2013) y las dataciones en el margen del Pauli sa Ussa. En los cortes descritos, se reconoce un estrato superficial que puede tener entre varios decímetros y en torno a $1.2 \mathrm{~m}$ de potencia, de arenas sueltas de color marrón claro a gris, ya sea sobre arenas más antiguas a menudo de tono rojizo (Bau Arena), sobre material fluvial como en Pauli Stincus (Nicosia et al., 2013), o en el margen de humedales (Pauli sa Ussa). En este último punto hay una capa de arenas de alrededor de un metro de potencia por encima de los niveles púnicos (siglo $\mathrm{V}$ a.C.). Por otra parte, en otros sectores como en la costa atlántica andaluza, los episodios holocenos de acumulación eólica sólo se desencadenan después del 2700 cal B.P., cuando se inicia la progradación en la costa de flechas y dunas asociadas (Borja et al., 1997; Borja et al., 1999; Zazo et al., 2011). Esto se explica según dichos autores porque anteriormente gran parte de la arena queda atrapada en los estuarios durante su fase agradacional. También podría introducirse en la discusión el efecto del episodio climático de la edad de hierro (desde el siglo VIII a.C.) detectado en las cuencas fluviales mediterráneas (Gutiérrez y Peña, 1998) y en las llanuras de inundación costeras, que aportó gran cantidad de material arenoso fluvial a la costa (Carmona y Ruiz, 2011). Un nuevo episodio eólico se produjo durante el pico frío de la P.E.H. en el siglo XVII. Durante dicho episodio se produjo el crecimiento de flechas y dunas y la progradación de deltas en diversos puntos de la costa mediterránea (Caputo et al., 1987; Bellotti, 2000; Amorosi y Milli, 2001; Anthony et al., 2014).

Por otro lado, en el caso de Terralba, es necesario valorar el efecto de la eliminación de la cobertura vegetal para la puesta en cultivo de las dunas. Es muy probable que la deforestación de las antiguas dunas en torno a las granjas púnicas desencadenara episodios de reactivación eólica de las arenas que pueden haber conformado el estrato superior. Este nivel histórico contiene abundantes restos arqueológicos y en el corte cercano a Pauli sa Ussa entierra el ambiente de humedal contemporáneo a época púnica. Asimismo, la remoción y reactivación eólica de estas superficies deforestadas es una importante variable en la formación de áreas de deflación eólica $y$, a su vez, en la aparición de multitud de pequeñas depresiones endorreicas o humedales ("paulis").

Tal y como hemos destacado, precisamente en estos ambientes en los que alternan alineaciones arenosas (dunas) y pequeñas depresiones o "paulis" es 


\section{PUNTO 26 DELTA DEL MOGORO}

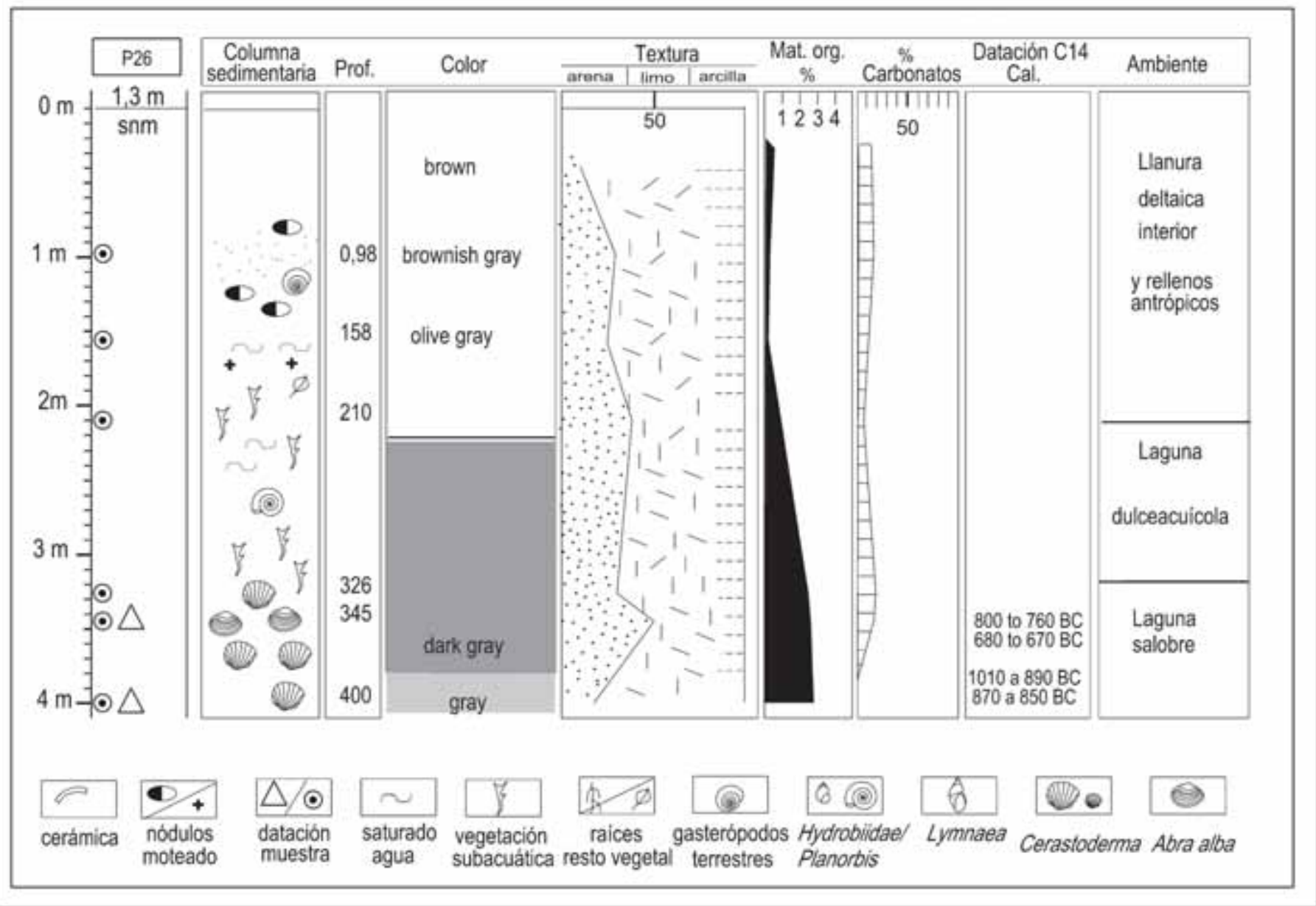

Figura 10. Registro sedimentario del lagoon-delta del río Mogoro. Figure 10. Sedimentary record of the Mogoro river lagoon delta.

donde se concentra la mayor parte de granjas púnicas en la llanura de Terralba. Puede explicarse tal concentración por factores edáficos (Nicosia et al., 2013) y la disponibilidad de agua dulce en el entorno del Pauli sa Ussa. Dos pozos de forma oblonga de $1.40 \mathrm{~m}$ de profundidad que llegaban a la capa freática entonces existente, mucho más superficial que en la actualidad, se encontraron en perfecto estado en el yacimiento Truncu e Molas (Van Domlemen et al., 2007). En la actualidad, el encauzamiento y desvío de cauces, las bonificaciones y la puesta en regadío a partir del canal delTirso han alterado completamente la alimentación de la capa freática en la llanura deTerralba y han provocado incluso la intrusión del acuífero salino en el litoral (Cau et al., 2002). Después del desvío y revestimiento con hormigón del río Mogoro se secaron la mayoría de pozos (Barrocu, 2004).
Cambios en la línea de costa: el relleno de los ambientes lagunares y la progradación del delta del río Tirso

En cuanto al relleno de los espacios de lagoon costeros, según los registros sedimentarios y las dataciones realizadas en el sondeo del delta interior del río Mogoro se deduce que el lagoon di Sassu en época fenicia y púnica se extendía bastante más hacia el interior. La abundancia de Cerastoderma sp. en la base del sondeo indica un nivel de salinidad fácilmente explicable si el lagoon di Sassu estuviera abierto al mar todavía en época púnica. Antes de la progradación de la flecha meridional más reciente del Tirso, el agua marina invadiría completamente el lagoon. El cierre de s'Ena Arrubia, el Pauli Longu di Sassu y el Pauli Pirastu (Figs. 4 y 5 ) se habría confor- 


\section{PUNTO 13 MARISMA NEAPOLIS}

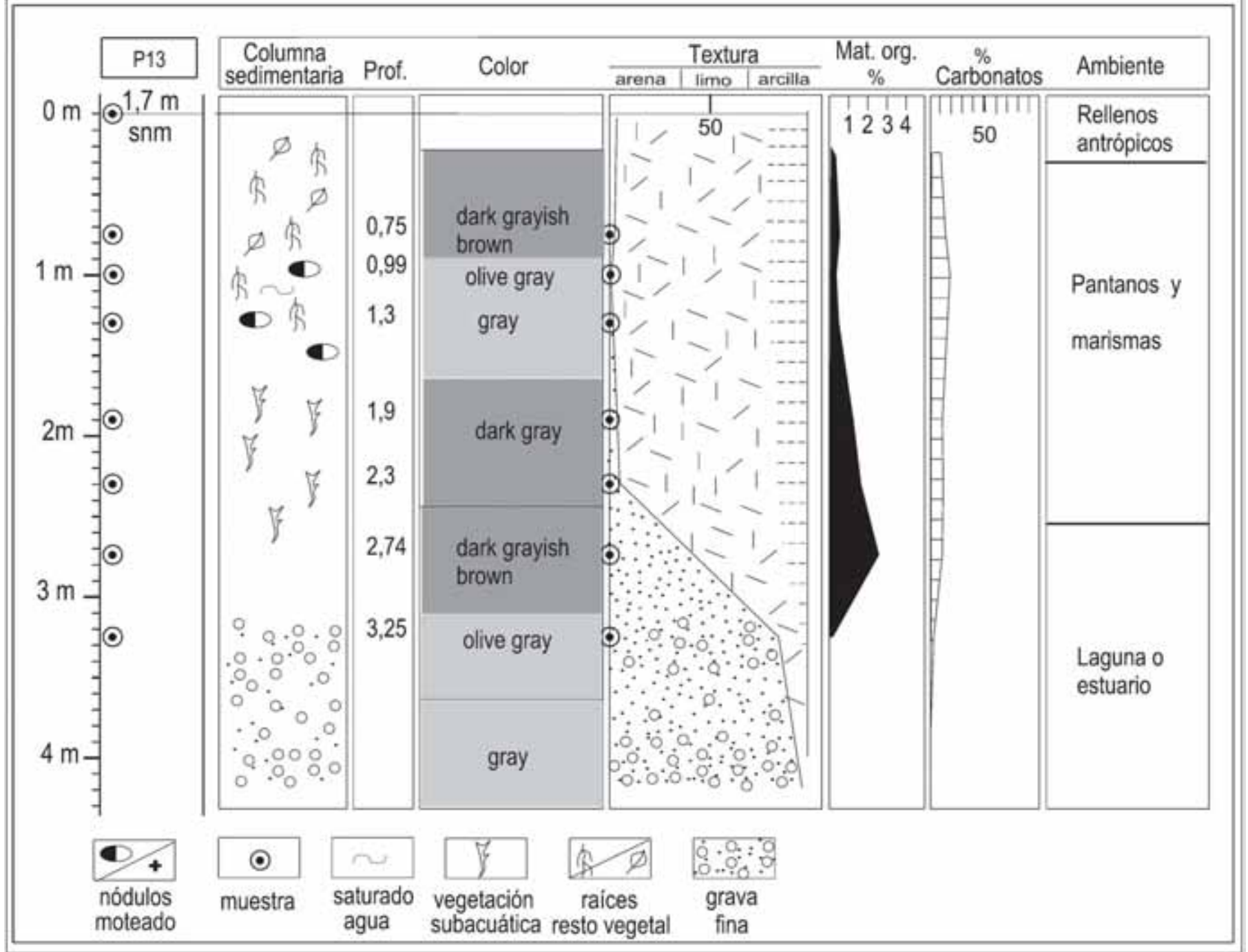

Figura 11. Registro sedimentario de Sta. Maria di Neapolis.

Figure 11. Sedimentary record of the Sta. Maria di Neapolis.

mado en época posterior. Teniendo en cuenta que la parte inferior del registro son sedimentos naturales de lagoon salobre y deltaicos es muy posible que las embarcaciones pudieran penetrar por el lagoon di Sassu hasta las proximidades de Terralba o al menos hasta los pequeños resaltes cercanos. De igual forma, cabe pensar en un considerable aumento de la extensión del lagoon hacia el interior de Neapolis, hasta donde se debía poder llegar fácilmente con embarcaciones en época púnica-romana.

Así, un factor decisivo en la evolución de la línea de costa son los aportes sedimentarios del río Tirso. Sucesivas barreras arenosas, cada vez más avanza- das hacia el mar, conformaron un delta de oleaje en los últimos milenios. La posición de un yacimiento nurágico (Edad del Bronce) sobre uno de estos cordones al sur delTirso, a $2500 \mathrm{~m}$ de la línea de costa, aporta una fecha mínima de la progradación (Fig. 4). Los cambios producidos son acordes con los descritos en llanuras costeras mediterráneas donde muchos puertos antiguos ubicados en la línea de costa o en el interior de lagoons quedaron sin acceso al mar a causa de la progradación de cuerpos arenosos en los frentes deltaicos (Marriner y Morhange, 2007; Anthony et al., 2014; Morhange et al., 2015). Un ejemplo de yacimiento fenicio-púnico accesible 
Ruiz, J.M., et al., 2018. Geomorfología y cambio ambiental en el entorno de los... Boletín Geológico y Minero, 129 (1/2): $331-351$

desde la costa es Lixus, con puerto en el actual estuario (mareal) colmatado del río Loukkos (Carmona y Ruiz, 2009). Otros muchos ejemplos se pueden citar en la Andalucía mediterránea, donde numerosos puertos fenicio-púnicos se ubicaron en lagoons que fueron posteriormente rellenados por sedimentos en las desembocaduras fluviales (Schulz, 1983; Hoffmann, 1988 y 1995; Schubart, 1991; Arteaga y Schulz, 1997; Carmona, 1999; Senciales y Malvárez, 2003).

\section{Conclusiones}

Los resultados de este trabajo aportan abundantes datos sobre el contexto ambiental de la colonización púnica en la llanura costera de Terralba, al tiempo que se hallaron evidencias de significativos cambios geomorfológicos y ambientales globales durante más de dos milenios que afectaron a la línea de costa, lagoons, depresiones palustres y a los ambientes fluviodeltaico y eólico. Entre estos cambios hay que destacar el desarrollo de flechas litorales longitudinales en línea de costa, la colmatación de lagoons y "paulis" y la redistribución eólica de la arena sobre amplias superficies. Por un lado, se produjo el paulatino relleno de los grandes lagoons salobres costeros con sedimentos aportados por deltas interiores. Por otro lado, los procesos eólicos han continuado remodelando las superficies arenosas de la llanura de Terralba durante los últimos milenios. Un manto eólico reciente de potencia variable (entre varios decímetros y algo más de $1 \mathrm{~m}$ ) recubre buena parte de la llanura. De forma paralela los procesos de deflación eólica pueden explicar el origen de muchas de las pequeñas depresiones endorreicas en la llanura.

Desde el punto de vista geoarqueológico, la gran concentración de granjas púnicas en la llanura de Terralba sobre los mantos eólicos se puede relacionar por factores edáficos (Nicosia et al., 2011) e hidrogeológicos locales. Las condiciones del suelo eran muy adecuadas para el crecimiento de cultivos arbustivos, además de facilitar el drenaje del exceso de agua en invierno. Por otra parte, si se tiene en cuenta la fuerte y prolongada sequedad estival que caracteriza el clima de la zona, el afloramiento de agua dulce en los paulis más continentales y sobre todo el cortejo de humedales en torno al Pauli sa Ussa pudo ser un factor determinante en el asentamiento de las numerosas granjas púnicas que allí se localizan. Por el contrario, la franja más próxima a la costa estaba formada por una dispersión de pequeños lagoons salobres que alternan con cordones dunares, con peores condiciones para el establecimiento de explo- taciones agropecuarias. De hecho toda esta franja de terreno no fue colonizada en época púnica y permaneció sin población hasta que fue totalmente transformada para el regadío con la "Bonifiche sarda". Finalmente, respecto a la posible comunicación costera de estos asentamientos a través de puertos en lagoons en época púnica, el registro sedimentario del delta del río Mogoro confirma que el lagoon de Sassu mediados del primer milenio a.C. se extendía hasta cerca de Terralba. Por último, consideramos que resultaría clave un estudio detallado de las fases holocenas de la progradación del delta del río Tirso (con dataciones absolutas) para la reconstrucción del paleopaisaje arqueológico de la llanura costera de Terralba.

\section{Agradecimientos}

Agradecemos todos los comentarios efectuados por los dos revisores anónimos, así como también las sugerencias y revisión de los editores que sin duda han servido para mejorar la calidad del trabajo. Agradecemos al Sr. Alberto Manca (Agronomo de la Agenzia regionales per I'Agricoltura) habernos facilitado una copia digital de la "Planimetria della Tenuta della Societa Bonifiche Sarde di Terralba, escala original 1:10000". Agradecemos al Sr. Graziano Fadda habernos facilitado fotografías y copias digitales de la cartografía histórica (mapas topográficos) del "Archivio Storico della Società Bonifiche Sarde S.p.A". Este trabajo ha sido financiado por el proyecto: Cerdeña e Ibiza: La organización rural en época púnica. Ministerio de Economía y Competitividad HAR2012- 32488. Los trabajos de campo de índole geomorfológica y edafológica fueron financiados con ayuda de National Geographic Exploration. También se ha dispuesto de financiación del proyecto: CSO 2012-32367. Procesos y cambios hidrogeomorfológicos en llanuras de inundación costeras mediterráneas ante la variabilidad climática y la acción humana. Una aproximación multiescalar. Dirección General de Investigación Científica y Técnica. Finalmente, este trabajo ha dispuesto de dataciones $\mathrm{C} 14$ realizadas en el ámbito del proyecto $A^{*}$ MIDEX ( $n^{\circ}$ ANR-11-IDEX0001-02), perteneciente al programa del Gobierno de Francia "Investissements d'Avenir" (French National Research Agency)".

\section{Referencias}

Amorosi, A. and Milli, S. 2001. Late Quaternary depositional architecture of Po and Tevere river deltas (Italy) and 
worldwide comparison with coeval deltaic successions. Sedimentary Geology, 144, 357-375.

Andreucci, S., Pascucci, V. and Clemmensen, L.B. 2006. Upper Pleistocene coastal deposits of West Sardinia: a record of sea level and climate change. GeoActa, 5, 7996.

Andreucci, S., Pascucci, V., Murray, A.S. and Clemmensen, L.B. 2009. Late Pleistocene coastal evolution of San Giovanni di Sinis (west Sardinia, Western Mediterranean), Sedimentary Geology, 216, 104-116.

Andreucci, S., Clemmensen, L.B. and Pascucci, V. 2010. Transgressive dune formation along a cliffed coast, NW Sardinia (Italy): a record of Late Pleistocene climate change. In: Late Pleistocene response to climate change and sea level variation. Terra Nova, 22, 424-433.

Andreucci, S., Clemmensen, L.B., Murray, A.S. and Pascucci, V. 2010. Middle to late Pleistocene coastal deposits of Alghero, northwest Sardinia (Italy): chronology and evolution, Quaternary International, 222, 3-16.

Andreucci, S., Bateman, M.D., Zucca, C., Kapur, S., Ak it, I., Dunajko, A. and Pascucci, V. 2012. Evidence of Saharan dust in upper Pleistocene reworked palaeosols of Northwest Sardinia, Italy: palaeoenvironmental implications. Sedimentology, 59, 917-938.

Angelone, M., Gasparini, C., Guerra, M., Lombardi, S., Pizzino, L. Quattrocchi, F., Sacchi E. and Zuppi G.M. 2005. Fluid geochemistry of the Sardinian Rift-Campidano Graben (Sardinia, Italy): fault segmentation, seismic quiescence of geochemically "active" faults, and new constraints for selection of $\mathrm{CO} 2$ storage sites. Applied Geochemistry, 20, 317-340.

Anthony, E., Marriner, N. and Morhange, Ch. 2014. Human influence and the changing geomorphology of Mediterranean deltas and coasts over the last 6000 years: From progradation to destruction phase?. EarthScience Reviews, 139, 336-361.

Arteaga, O. and Schulz, H. D. 1997. El puerto fenicio de Toscanos. Investigación geoarqueológica en la costa de la Axarquía (Vélez-Málaga 1983-84). In: Aubet, M. E. (ed.), Los fenicios en Málaga. Servicio de Publicaciones, Universidad de Málaga, 87-154.

Barrocu, G. 2004. Stop 4.3. From Monte Arborone to Arborea. Hydrogeology of the Island of Sardinia (Italy). $32^{\text {nd }}$ International Geological Congress. Vol 5, pp 35-54.

Beccaluva, L., Deriu M., Macciotta, G., Savelli, C. and Venturelli, G. 1977. Geochronology and magmatic character of the pliocene-pleistocene volcanism in Sardinia (Italy). Bulletin Volcanologique, vol. 40 (3), 153-168.

Bellotti, P., Caputo, C., Davoli, L., Evangelista, S., Garzanti, E., Pugliese, F. and Valeri, P. 2004. Morpho-sedimentary characteristics and Holocene evolution of the emergent part of the Ombrone River delta (southern Tuscany). Geomorphology, 61, 71-90.

Belluomini, G., Branca M., Delitalia L., Pecorini, G. and Spano, C. 1986. Isoleucine Epimerization Dating of Quaternary Marine Deposits in Sardinia, Italy. Zeitschrift für Geomorphology, 62, 109-117.

Belluomini, G. and Delitala, L. 1988. Amino acid racemization dating of quaternary deposits of central and Southern Italy. Organic Geochemistry, 13, 4-6, 735-740.
Borja, F., Gutiérrez, J.M. and Díaz del Olmo, F. 1997. Fases de dunas durante el Holoceno reciente en el litoral del Puerto de Santa María (Cádiz). Geogaceta, 21, 39-42.

Borja, F., Zazo, C., Dabrio, C.J., Díaz del Olmo, F., Goy, J.L. and Lario, J. 1997. Holocene aeolian phases and human settlements along the Atlantic coast of southern Spain. The Holocene, 9 (3), 333-339.

Caputo, C., La Monica, G.B., Lupia Palmieri, E. and Pugliese, F. 1987. Physiographic characteristics and dynamics of the shores of Rome (Italy). In: Gardiner, V. (Ed.), Proceeding of the First International Conference on Geomorphology, Part I. Wiley Chichester, pp. 1185-1198.

Carmignani L., Oggiano G., Barca S., Conti P., Salvadori, I., Eltrudis, A., Funedda, A. and Pasci, S. 2001. Note illustrative della Carta Geologica della Sardegna a scala 1:200.000. Memorie Descrittive della Carta Geologica d'Italia, 60, 283 pp.

Carmona, P. 1999. Evolución paleogeográfica y geomorfológica del entorno del Cerro del Villar. In: Aubet, M.E., Carmona, P., Curià, E., Delgado, A., Fernández Cantos, A. and Párraga, M. (eds.), Cerro del Villar I. El asentamiento fenicio en la desembocadura del río Guadalhorce y su interacción con el hinterland. Arqueología Monografías, Junta de Andalucía, 33-41.

Carmona, P. and Ruiz, J.M. 2009. Geomorphological Evolution of the River Loukkos Estuary Around the Phoenician City of Lixus on the Atlantic Littoral of Morocco. Geoarchaeology: An International Journal,24 (6), 821-845.

Carmona, P. and Ruiz, J.M. 2011. Historical morphogenesis of the Turia River coastal flood plain in the Mediterranean littoral of Spain. Catena, 86, 139-149.

Casula, G., Cherchi, A., Montadert, L. Murru, M. and Sarria, R. 2001. The Cenozoic graben system of Sardinia (Italy): geodynamic evolution from new seismic and field data. Marine Petroleum Geology, 18, 863-888.

Cau, P., Lecca, G., Muscas, L. Barrocu, G. and Uras, G. 2002. Seawater intrusión in the plain of Oristano (Sardinia, Italy). $17^{\text {th }}$ Salt Water Intrusion Meeting, Delft, The Netherlands, 435-444.

Cherchi, E. and Montadert, L. 1982. Oligo-miocene rift of Sardinia and the early history of the Western Mediterranean Basin. Nature, 298, 736-739.

Díes, E., Van Dommlemen, P. y Gómez Bellard, C. 2010. Excavaciones en la granja púnica de Pauli Stincus (Terralba, Cerdeña). Saguntvm. Papeles del Laboratorio de Arqueología de Valencia, 42, 123-127.

Ente Autonomo Del Flumendosa 1998. Nuovo studio dell'idrologia superficiale della Sardegna. Regione Autonoma della Sardegna. Assessorato della Programmazione, Bilancio ed Assetto del Territorio, Centro Regionale di Programmazione, Cagliari, CD-rom.

Fais, S., Klingele, E.E. and Lecca, L. 1996. Oligo-Miocene half graben structure in Western Sardinian Shelf (Western Mediterranean). Reflection seismic and aeromagnetic data comparison. Marine Geology, 133, 203-222.

Gutiérrez, M. and Peña, J.L., 1998. Geomorphology and late Holocene climatic change in Northeastern Spain. Geomorphology, 23, 205-217. 
Ruiz, J.M., et al., 2018. Geomorfología y cambio ambiental en el entorno de los... Boletín Geológico y Minero, 129 (1/2): $331-351$

Hoffmann, G. 1988. Holozänstratigraphie und küstenlinienverlangerung an der Andaluischen mittelmeerküste, Berichte aus dem fachbereich Geowissenschaften der Universität Bremen, 2, Allemagne, $174 \mathrm{p}$.

Henningsen, D., Kelletat, D. and Hagn, H. 1981. Die quartären Aolianite von Ibiza und Formentera (Balearem, Mittelmeer) und ihre Bedeutung für die Entwicklungsgeschivhte der Inseln. Eiszeitalter $u$. Gegenwart, 31, 109-133.

Hoffmann, G. 1995. Die nacheiszeitliche Landschaftsentwicklung der andalusischen Mittelmeerküste am Beispiel del phönizischen Niederlassung Sexi (Almuñécar), Madrider Mitteilungen, 36, 191-198.

Lecca, L. and Carboni, S. 2007. The Tyrrhenian section of San Giovanni di Sinis (Sardinia): stratigraphic record of an irregular single high stand. Rivista Italiana di Paleontologia e Stratigrafia, 113, 509-523.

Magni, P., Como, S., Cucco, A., De Falco, G., Domenici, P., Ghezzo, M., Lefrançois, C., Simeone, S. and Perilli, A. 2008. A Multidisciplinary and Ecosystemic Approach in the Oristano Lagoon-Gulf System (Sardinia, Italy) as a Tool in Management Plans. Transitional Waters Bulletin, 2 (2), 41-62.

Marriner, N. and Morhange, C. 2007. Geoscience of ancient Mediterranean harbours. Earth-Science Reviews, 80 (34), 137-194.

Morhange, Ch., Marriner, N., Baralis, A., Blot, M.L., Bony, G., Carayon, N., Carmona, P., Flaux, C., Giaime, M., Goiran, J.P., Kouka, M., Lena, A., Oueslati, A., Pasquinucci, M. and Porotov, A. 2015. Dynamiques géomorphologiques et typologie géoarcheologique des ports antiques en contextes lagunaires. Quaternaire, Revue de L'Association française pour l'étude du Quaternaire (AFEQ) France, 26 (2), 117-139.

Nicosia, C., Langohr, R., Carmona, P., Gómez Bellard, Modrall, E., Ruiz, J.M. and Van Dommelen, P. 2013. Land Use History and Site Formation Processes at the Punic Site of Pauli Stincus in West Central Sardinia, Geoarchaeology, 28, 373-393.

Pala, A., Pecorini, G., Porcu, A. and Serra, S. 1982. Schema geologico strutturale della Sardegna. In: Ricerche Geotermiche in Sardegna con particolare riferimento al Graben del Campidano. CNR-PFE-SPEG-RF10, CNR-Pisa, 7-24.

Pérez Jordà, G., Morales Pérez, J.V., Marlasca, R., Gómez Bellard, C. and van Dommelen, P. 2010. La alimentación en una granja púnica de Cerdeña, Sagvntvm Extra, 9, 295-302.

Reimer, P.J., Baillie, M.G.L., Bard, E., Bayliss, A., Beck, J.W., Bertrand, C.J.H., Blackwell, P.G., Buck, C.E., Burr, G., Cutler, K.B., Damon, P.E., Edwards, R.L., Fairbanks, R.G., Friedrich, M., Guilderson, T.P., Hogg, A.G., Hughen, K.A., Kromer, B., McCormac, G., Manning, S., Bronk Ramsey, C., Reimer, R.W., Remmele, S., Southon, J.R., Stuiver, M., Talamo, S., Taylor, F.W., van der Plicht, J. and Weyhenmeyer, C.E. 2004. INTCAL04 Terrestrial Radiocarbon Age Calibration, 0-26 Cal Kyr BP. Radiocarbon, 46 (3), 1029-1058.

Rohdenburg, H. 1992. Nouvelles données du 14C provenant du Maroc et de I'Espagne et leur apport pour la connaissance du développement du relief et la pédogenese dans I'Holocene et le pédogenese dans I'Holocene et le Pleistocene récent. Cuaternario y Geomorfología, 6, 157170.

Sevinek, J. and Kummer, E. A. 1984. Eolian Dust Deposition on the Giara Di Gesturi basalt Plateau, Sardinia. Earth Surface Processes and Landforms, 9, 357-364.

Soru, M.C. 2000. Terralba: una bonifica senza redenzione: origini, percorsi, esiti. Studi storici Carocci, 5, Roma, 409 p.

Schubart, H. 1991. Investigaciones geológicas y arqueológicas sobre la relación costera de los asentamientos fenicios en la Andalucía Mediterránea. In: Atti del II Congreso Internazionale di Studi Fenici e Punici, Consiglio Nazionale delle Ricerche, Istituto per la civilitá fenicia e púnica. Roma, 1245-1251.

Schulz, H. D. 1983. Zur lage Holozäner Küsten in den Mündungsgebieten des río de Vélez und des río Algarrobo (Málaga), Madrider Mitteilungen, 24, 59-64.

Senciales, J. M. and Malvárez, G. 2003. La desembocadura del río Vélez (provincia de Málaga, España). Evolución reciente de un delta de comportamiento mediterráneo, Cuaternario y Geomorfología, 17 (1-2), 47-61.

Spano, C., Belluomini, G., Carboni, S., Congiu, A.C., Murgia, D., Tomassi, A. V. and Vesica, P. 2005. A synthesis of the Tyrrhenian in Sardinia (Italy). Correlations, biocenoticbatimetric and palaeoenvironmental aspects. VI Reunión de Cuaternario Ibérico, Libro de Actas (Gibraltar, septiembre 2005), 50-52.

Ulzega, A. and Hearty, A. 1986. Geomorphology, stratigraphy and geochronology of late quaternary marine deposits in Sardinia. Zeitschrift für Geomorphologie, 62, 119-129.

Van Dommelen, P. 1998. On colonial grounds. A comparative study of colonialism and rural settlement in 1st millennium B.C. west central Sardinia. Archaeological Studies Leiden University, 2. Faculty of Archaeology, Leiden University, Leiden: 296 p.

Van Dommelen, P. 2003. Insediamento rurale ed organizzazione agraria nella Sardegna centro-occidentale, In: C. Gómez Bellard (ed.), Ecohistoria del paisaje agrario. La agricultura fenicio-púnica en el Mediterráneo, Universitat de València, Valencia: 129-149.

Van Dommelen, P., Sharpe, L., and McLellan, K. 2006. Insediamento rurale nella Sardegna punica: il progetto Terralba (Sardegna). In: Akerraz, A., Ruggeri, P., Siraj, A. and Vismara, C. (eds.), L'Africa romana. Mobilità delle persone e dei popoli, dinamiche migratorie, emigrazioni ed immigrazioni nelle province occidentali dell'Impero romano, Atti del XVI convegno di studio, Rabat, 15-19 dicembre 2004. L'Africa romana,16. Carocci, Rome, 153173.

Van Dommelen, P., Gómez Bellard, C. and Tronchetti, C. 2007. La excavación de la granja púnica de Truncu 'e Molas (Terralba, Cerdeña). Sagvntvm. Papeles del Laboratorio de Arqueología de Valencia, 39, 179-184.

Van Dommelen, P., Gómez Bellard, C. and Tronchetti, C. 2012. Insediamento rurale e produzione agraria nella Sardegna punica: la fattoria di Truncu 'e Molas (Terralba, OR). In: Del Vais, C. (ed.), Epi Oinopa Ponton, Studi sul 
Mediterraneo antico, in ricordo di Giovanni Tore. Università degli Studi di Cagliari, 501-516.

Van Dommelen, P., Gómez Bellard, C. Pérez Jordà, G. and Vendrell Betí, A. (eds.) (in press). Truncu'e Molas (Terralba, Oristano): un asentamiento rural en la Cerdeña púnica, Sagvntvm Extra, n 17, Valencia.
Zazo, C., Dabrio, Goy, J.L., C.J., Borja, F., Lario, J., Roquero, T., Bardají, T., Cabero, A., Polo, M.D. and Borja, C. 2011. EI complejo eólico de El Abalorio (Huelva). In: Sanjaume, E. and García Prieto, F.J. (coord.), Las dunas en España. Sociedad Española de Geomorfología, Puerto Real (Cádiz), 407-425.

Recibido: marzo 2015

Revisado: junio 2015

Aceptado: julio 2015

Publicado: marzo 2018 
